\title{
Schur flexibility of cominuscule Schubert varieties C. Robles
}

Let $X=G / P$ be a cominuscule rational homogeneous variety. (Equivalently, $X$ admits the structure of a compact Hermitian symmetric space.) We say a Schubert class $\xi$ is Schur rigid if the only irreducible subvarieties $Y \subset X$ with homology class $[Y] \in \mathbb{Z} \xi$ are Schubert varieties. Robles and The identified a sufficient condition for $\xi$ to be Schur rigid. In this paper, we show that the condition is also necessary.

$\begin{array}{lll}1 & \text { Introduction } & \mathbf{9 8 0}\end{array}$

$\begin{array}{lll}1.1 & \text { History } & 982\end{array}$

$\begin{array}{lll}1.2 & \text { Contents } & 982\end{array}$

2 Rigid classes in the irreducible $G / P \quad 983$

Key to Figures 1 and $2 \quad 984$

2.1 Quadric hypersurfaces $\quad 984$

$\begin{array}{lll}2.2 & \text { Grassmannians } & 987\end{array}$

$\begin{array}{lll}2.3 & \text { Lagrangian Grassmannians } & 987\end{array}$

$\begin{array}{lll}2.4 & \text { Spinor varieties } & 988\end{array}$

$\begin{array}{lll}3 & \text { Review } & 989\end{array}$

$\begin{array}{ll}3.1 & \text { Notation and background }\end{array}$

$\begin{array}{lll}3.2 & \text { The Schur differential system } & 991\end{array}$

$\begin{array}{ll}3.3 & \text { The Schubert differential system }\end{array}$ 
3.4 The characterization of Schubert varieties

3.5 Divisors

$4 \quad$ Flexibility

4.1 Outline of the proof of Theorem 4.1

4.2 Proof of Theorem 4.1 in the case that $H_{1}$ fails

4.3 Proof of Theorem 4.1 in the case that $\mathrm{H}_{2}$ fails

1001

Acknowledgments

1003

Appendix A Geometric versus representation theoretic descriptions

A.1 Notation

A.2 Odd-dimensional quadrics $Q^{2 n-1}=B_{n} / P_{1}$ 1003

A.3 Even-dimensional quadrics $Q^{2 n-2}=D_{n} / P_{1}$ 1004

A.4 Grassmannians $\operatorname{Gr}(i, n+1)=A_{n} / P_{i}$ 1005

Partitions versus a, J 1006

A.5 Lagrangian Grassmannians $\operatorname{LG}(n, 2 n)=C_{n} / P_{n} \quad 1007$ Partitions versus a, J 1008

A.6 Spinor varieties $\mathcal{S}_{n}=D_{n} / P_{n}$ 1009

Partitions versus a, J 1010

References 1011

\section{Introduction}

Let $X=G / P$ be a rational homogeneous variety. The Schubert classes form an additive basis of the integral homology $H_{\bullet}(X, \mathbb{Z})$. In 1961 , Borel and 
Haefliger [2] asked: given a Schubert class $\xi_{w}$ represented by a Schubert variety $Y_{w}$, aside from the $G$-translates $g \cdot Y_{w}$, are there any other algebraic representatives of the Schubert class? In some cases, the Schubert varieties are the only algebraic representatives, and we then say the Schubert class is rigid. This problem has been studied by several people, including Hartshorne et al. [9], Bryant [3], Walters [21], Hong [11, 12], Robles and The [19] and Coskun $[6,7]$.

It is a striking consequence of B. Kostant's work [16, 17] that, when $X$ is cominuscule, ${ }^{1}$ the varieties $Y \subset X$ that are homologous to an integer multiple of the Schubert class $\xi_{w}$ (that is, $[Y] \in \mathbb{Z} \xi_{w}$ ) are characterized by a system of differential equations known as the Schur system; cf. Section 3.2. The Schubert varieties $\left\{g \cdot Y_{w} \mid g \in G\right\}$ are the trivial solutions. When there exist no non-trivial, irreducible solutions, we say that the Schubert class is Schur rigid. Every Schur rigid $\xi_{w}$ is rigid, and this provides a differential-geometric approach to the algebro-topological question above.

Associated to the differential system is a Lie algebra cohomology. The cohomology contains a distinguished subspace $\mathcal{O}_{w}$. It is known [19] that the Schubert class $\xi_{w}$ is Schur rigid when $\mathcal{O}_{w}=0$; we say that the space $\mathcal{O}_{w}$ is a cohomological obstruction to rigidity. The goal of this paper is to show the converse: when $\mathcal{O}_{w}$ is non-trivial, there exist non-trivial solutions $Y$. Precisely, $Y$ is an irreducible subvariety of $X$ such that (i) $[Y] \in \mathbb{Z} \xi_{w}$, and (ii) $Y$ is not a $G$-translate of $Y_{w}$; this is Corollary 4.3 .

From the perspective exterior differential systems (EDS) this construction of non-trivial solutions is very interesting. It is a relatively common practice to establish rigidity through the machinery of EDS. In contrast, it is often considerably more difficult to construct non-trivial solutions. (A striking example is R. Bryant's construction of metrics with exceptional holonomy $G_{2}$ and $\operatorname{Spin}(7)$ in [4].) Moreover, the construction is typically local. So, what is particularly interesting here (and I expect will have applications to other problems) is the use of Lie algebra cohomology to construct non-trivial, global (they are algebraic varieties) solutions.

While the construction of the non-trivial solutions $Y$ (in the proof of Theorem 4.1) is explicit, it is in terms of representation theoretic data, and as a result is not geometrically transparent. Geometric (and explicit) constructions of $Y$ are given in [8], where a stronger (than Corollary 4.3)

\footnotetext{
${ }^{1}$ The Grassmannian $\operatorname{Gr}(k, n)$ of complex $k$-planes in $\mathbb{C}^{n}$ is an example of a cominuscule rational homogeneous variety (via the Plücker embedding).
} 
statement is proven: if $\mathcal{O}_{w} \neq 0$, then for any positive integer $m$ there exists an irreducible variety $Y$ representing $m \xi_{w}$.

\subsection{History}

Schur rigidity and the associated differential system were first studied independently by Walters [21] and Bryant [3]. Walters identified Schur rigid classes represented by (a) smooth Schubert varieties in $X=\operatorname{Gr}(k, n)$; and (b) codimension two Schubert varieties in $X=\operatorname{Gr}(2, n)$. Bryant identified Schur rigid classes represented by (a) smooth Schubert varieties in the cases that $X$ is the Grassmannian $\operatorname{Gr}(k, n)$ or the Lagrangian Grassmannian $\mathrm{LG}(n, 2 n)$; (b) the maximal linear subspaces in the classical cominuscule $X$; and (c) singular Schubert varieties of low (co)dimension in $\operatorname{Gr}(k, n)$. In the case that $Y_{w}$ is smooth, the results of Bryant and Walters, which were obtained case-by-case, were generalized to arbitrary cominuscule $X$ and given a beautiful, uniform proof by Hong [12]. There, the obstructions to rigidity are realized as a (Lie algebra) cohomological condition. Hong also showed that a large class of the singular Schubert varieties in the Grassmannian are Schur rigid [11]. Robles and The [19] extended the approach of [12] to give a complete list of the Schubert classes for which there exist no cohomological obstructions to rigidity: these classes are necessarily Schur rigid.

Several people have worked on the more restrictive problem of determining when $\xi_{w}$ is rigid, as well as the related problem of determining when $\xi_{w}$ admits a smooth representative; see $[6,7,9,10,13,14]$.

\subsection{Contents}

The main result of this paper is Theorem 4.1. The theorem yields Corollary 4.3, which is the result discussed above: if $\mathcal{O}_{w} \neq 0$, then the Schubert class $\xi_{w}$ is Schur flexible; that is, there exists an irreducible variety $Y$, which is not $G$-equivalent to $Y_{w}$, such that $[Y] \in \mathbb{Z} \xi_{w}$. As a corollary, we find that a Schubert class is Schur rigid if and only if its Poincaré dual is Schur rigid (Corollary 4.5).

In Section 2, we apply Corollary 4.3 to each of the classical (irreducible) cominuscule varieties in order to enumerate their Schur flexible classes (Theorems 2.1-2.4). In the case of the Lagrangian Grassmannian $\mathrm{LG}(n, 2 n)=C_{n} / P_{n}$ and the Spinor variety $\mathcal{S}_{n+1}=D_{n+1} / P_{n+1}$ we obtain the following corollary: there is an bijection between the set of Schubert classes in $\mathrm{LG}(n, 2 n)$, and the set of Schubert classes in $\mathcal{S}_{n+1}$. (This bijection 
preserves the partial order on the Hasse posets parameterizing the Schubert classes.) By Corollary 2.5, a Schubert class in $\mathrm{LG}(n, 2 n)$ is Schur rigid if and only if the corresponding class in $\mathcal{S}_{n+1}$ is Schur rigid.

The Schur rigid classes of the two exceptional (irreducible) cominuscule varieties are given in Figures 1 and 2.

\section{Rigid classes in the irreducible $G / P$}

The irreducible, cominuscule rational homogeneous varieties $X=G / P$ are:

○ the Grassmannian $\operatorname{Gr}\left(k, \mathbb{C}^{n}\right) \simeq \mathrm{SL}_{n}(\mathbb{C}) / P_{k}$ of $k$-planes in $\mathbb{C}^{n}$;

o the smooth quadric hypersurface $\mathcal{Q}^{m} \subset \mathbb{P}^{m+1} \simeq \mathrm{SO}(m+2) / P_{1}$;

○ the Lagrangian Grassmannian $\operatorname{LG}\left(n, \mathbb{C}^{2 n}\right)=\operatorname{Sp}_{2 n}(\mathbb{C}) / P_{n}$;

○ the Spinor variety $\mathcal{S} \simeq \operatorname{Spin}_{2 n}(\mathbb{C}) / P_{n}$;

$\circ$ the Cayley plane $E_{6} / P_{6}$, and the Freudenthal variety $E_{7} / P_{7}$.

The Grassmannians, quadrics, Lagrangian Grassmannians and Spinors, are the classical $X$; the Cayley plane and Freudenthal variety are the exceptional $X$.

Theorems 2.1-2.4 list the Schur rigid classes for each of the classical $X$ above. These theorems are proved as follows. By Corollary 4.3, a Schubert class $\xi_{w} \in H_{\bullet}(X, \mathbb{Z})$ is Schur rigid if and only if $\mathcal{O}_{w}=0$; equivalently, the condition $\mathrm{H}_{+}$(Definition 4.6) is satisfied. For each of the irreducible, cominuscule $X$, the Schubert classes satisfying the condition $\mathrm{H}_{+}$are given by [19, Theorem 6.1]. There, the Schubert classes are described in terms of representation theoretic data; cf. Section 3.4. This representation theoretic description is well-suited to the proof of Theorem 4.1, but is not geometrically transparent. The appendices give the translations between the representation theoretic descriptions and the familiar geometric descriptions for the classical $X$; see, in particular, Lemmas A.9, A.19 and A.27. These translations, applied to Corollary 4.3 and [19, Theorem 6.1], yield the geometric descriptions of the Schur rigid classes given by Theorems 2.2 to 2.4. Throughout,

$$
o=P / P \in G / P=X
$$

In the case of the two exceptional $X$, the Schubert classes satisfying the condition $\mathrm{H}_{+}$(equivalently, by Corollary 4.3, the Schur rigid classes) are given by [19, Tables 4 and 5]. This data is transcribed to Figures 1 and 2 (pages 7 and 8). 


\section{Key to Figures 1 and 2}

These figures are respectively the Hasse posets of the Cayley plane $E_{6} / P_{6}$ and Freudenthal variety $E_{7} / P_{7}$. Each node represents a Schubert class $\xi_{w}$ and is labeled with degree of $Y_{w}$ in the minimal homogeneous embeddings $E_{6} / P_{6} \hookrightarrow \mathbb{P} V_{\omega_{6}}$ and $E_{7} / P_{7} \hookrightarrow \mathbb{P} V_{\omega_{7}}$; cf. [1, Section 4.8]. (In fact, the Cayley plane and Freudenthal variety are also minuscule. So [1, Remark 4.8.4] may be used to compute the degree.) The height of the node indicates the dimension of $\xi_{w}$; in particular, the lowest node $o \in X$ is at height zero. Two nodes are connected if the Schubert variety associated with the lower node is a divisor of the Schubert variety associated with the higher node. The node is circled if the corresponding Schubert class is Schur rigid.

\subsection{Quadric hypersurfaces}

If the quadric hypersurface $\mathcal{Q} \subset \mathbb{P}^{2 m}$ is of odd dimension, then $[o]$ and $[\mathcal{Q}]$ are the only Schur rigid Schubert classes. This is seen by a simple topological argument, as follows. It is well-known that $H_{2 d}(\mathcal{Q}, \mathbb{Z})=\mathbb{Z}$ for all $0 \leq d \leq$ $2 m-1$; that is, $\xi_{w}$ spans $H_{2|w|}(\mathcal{Q}, \mathbb{Z})$ where, $|w|=\operatorname{dim}_{\mathbb{C}} \xi_{w}$. (See $[1$, p. 139 140] for an explicit description of the Schubert varieties; they are linear subspaces and degenerate quadrics.) Therefore, if $Y \subset \mathcal{Q}$ is any subvariety of dimension $d$ (for example, a general complete intersection), then $[Y] \in$ $\mathbb{Z}_{>0} \xi_{w}$, where $\xi_{w}$ is the unique Schubert class of dimension $d$. Thus, $\xi_{w}$ is Schur flexible, for all $\operatorname{dim}_{\mathbb{C}} \xi_{w} \neq 0,2 m-1$.

If the quadric $\mathcal{Q} \subset \mathbb{P}^{2 m+1}$ is of even dimension, then again $H_{2 d}(\mathcal{Q}, \mathbb{Z})=\mathbb{Z}$ for all $0 \leq d \leq 2 m$, except $d=m$. (See [1, pp. 142-143] for an explicit description of the Schubert varieties; again, they are linear subspaces and degenerate quadrics.) As above, the Schubert class $\xi_{w}$ is Schur flexible for all $\operatorname{dim}_{\mathbb{C}} \xi_{w} \neq 0, m, 2 m$. If $d=m$, then $H_{2 m}(\mathcal{Q}, \mathbb{Z})=\mathbb{Z} \oplus \mathbb{Z}$, and the two spanning Schubert classes $\xi_{w}$ and $\xi_{w^{\prime}}$ are each represented by a maximal linear space. These two Schubert classes are known to be Schur rigid by the work of Hong.

In summary, we have the following.

Theorem 2.1 (Schur rigidity in quadrics). (a) If the quadric $\mathcal{Q} \subset$ $\mathbb{P}^{2 m}$ is of odd dimension, then the only Schur rigid Schubert classes are $[o]$ and $[X]$.

(b) If the quadric $\mathcal{Q} \subset \mathbb{P}^{2 m+1}$ is of even dimension, then the Schur rigid Schubert classes are $[o],[X]$ and the two distinct classes $\xi_{w}, \xi_{w^{\prime}} \in H_{2 m}$ $(\mathcal{Q}, \mathbb{Z})$ represented by maximal linear subspaces. 


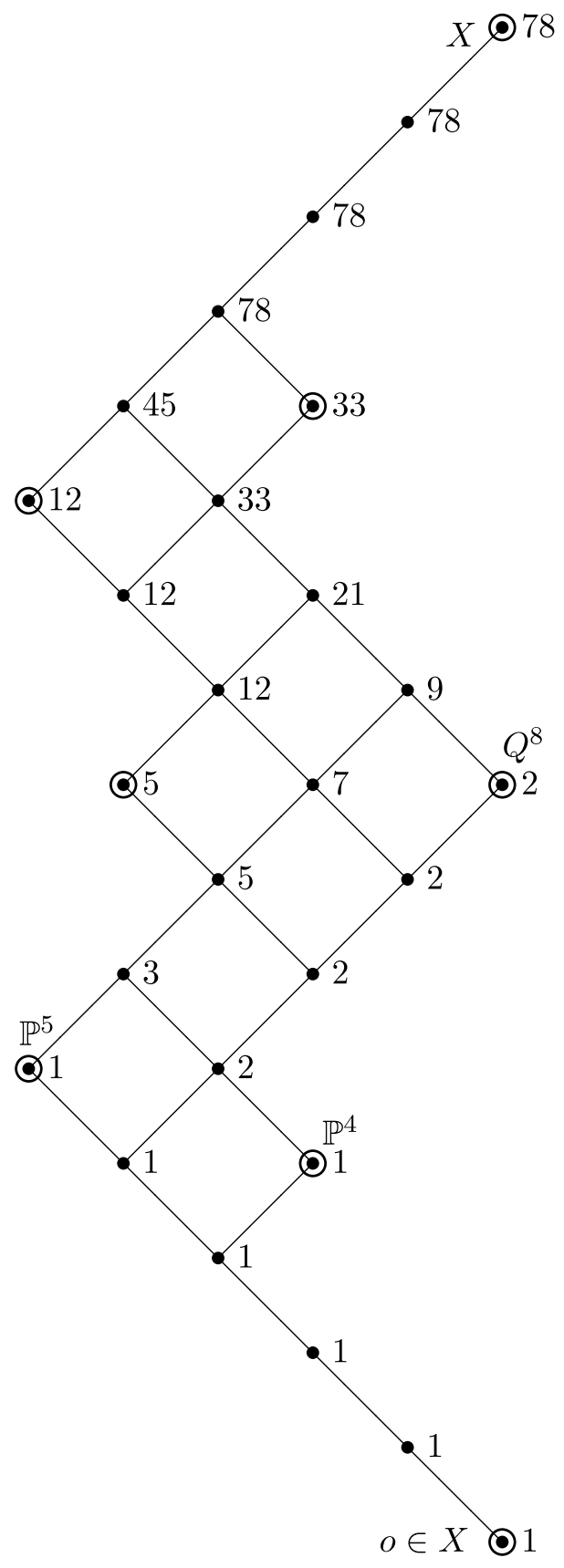

Figure 1: Hasse poset of the Cayley plane $E_{6} / P_{6}$. 


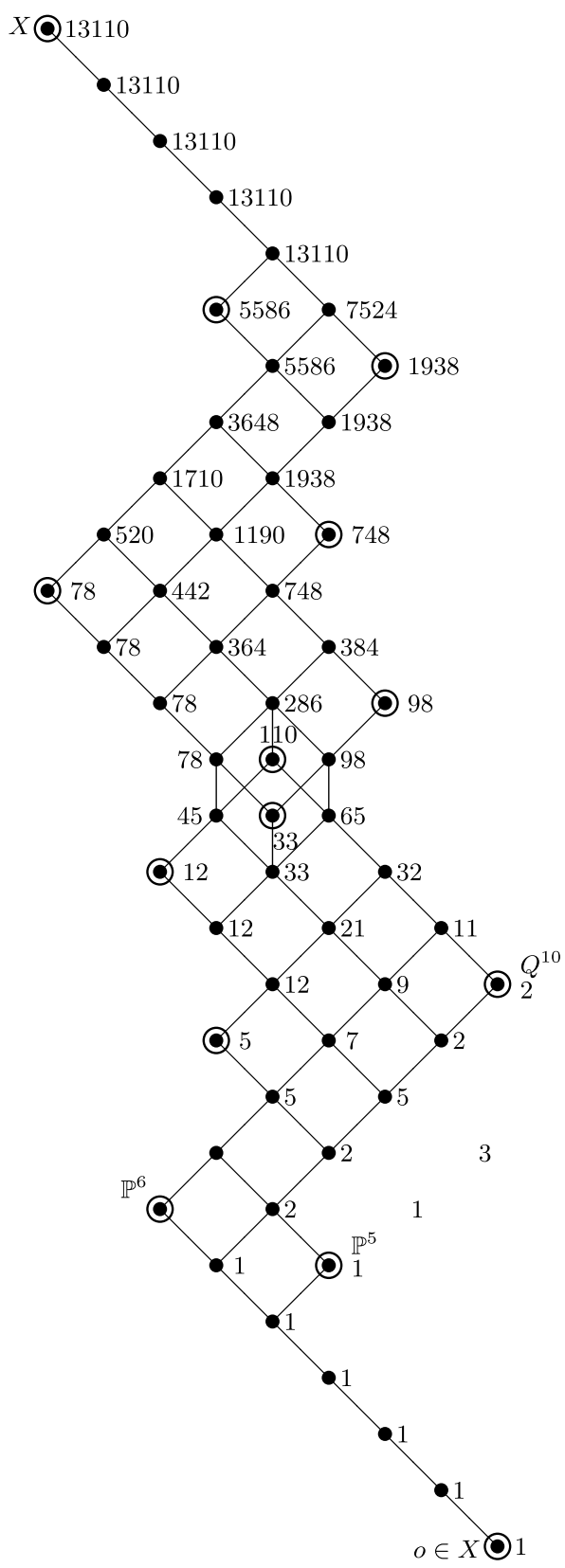

Figure 2: Hasse poset of the Freudenthal variety $E_{7} / P_{7}$. 


\subsection{Grassmannians}

It is well-known that Schubert varieties in $\operatorname{Gr}(\mathbf{i}, n+1)$ are indexed by partitions

$$
\lambda=\left(\lambda_{1}, \ldots, \lambda_{\mathrm{i}}\right) \in \mathbb{Z}^{\mathrm{i}} \quad \text { such that } 1 \leq \lambda_{1}<\lambda_{2}<\cdots<\lambda_{\mathrm{i}} \leq n+1
$$

cf. [1, Section 3.1.3]. Fix a flag $0 \subset F^{1} \subset F^{2} \subset \cdots \subset F^{n+1}=\mathbb{C}^{n+1}$. The corresponding Schubert variety is

$$
Y_{\lambda}\left(F^{\bullet}\right)=\left\{E \in \operatorname{Gr}(\dot{i}, n+1) \mid \operatorname{dim}\left(E \cap F^{\lambda_{k}}\right) \geq k, 1 \leq k \leq \mathbf{i}\right\}
$$

Define $\tilde{\lambda}_{k}=\lambda_{k}-k$; then $0 \leq \tilde{\lambda}_{1} \leq \cdots \leq \lambda_{i} \leq n+1-i$. Condense $\tilde{\lambda}$ by writing $\tilde{\lambda}=\left(\nu_{\mathrm{p}}^{c_{\mathrm{p}}}, \ldots, \nu_{1}^{c_{1}}, \nu_{0}^{c_{0}}\right)$ with $0 \leq \nu_{\mathrm{p}}<\cdots<\nu_{1}<\nu_{0} \leq n+1-\mathrm{i}$, and $0<c_{s}$, for all $0 \leq s \leq$ p. Corollary 4.3, [19, Theorem 6.1], Remark A.7 and Lemma A.9 yield the following.

Theorem 2.2 (Schur rigidity in Grassmannians). The Schubert class $\xi_{\lambda} \in H_{\bullet}(\operatorname{Gr}(\mathbf{i}, n+1), \mathbb{Z})$ is Schur rigid if and only if the following conditions hold:

(a) $\nu_{s-1}-\nu_{s} \geq 2$, for all $1 \leq s \leq \mathrm{p}$;

(b) $c_{s} \geq 2$, for all $1 \leq s \leq \mathrm{p}-1$;

(c) if $\nu_{\mathrm{p}}>0$, then $c_{\mathrm{p}} \geq 2$;

(d) if $\nu_{0}<n+1-i$, then $c_{0} \geq 2$.

\subsection{Lagrangian Grassmannians}

It is well-known that Schubert varieties in $\operatorname{LG}(n, 2 n)$ are indexed by partitions $1 \leq \lambda_{1}<\lambda_{2}<\cdots<\lambda_{n} \leq 2 n$ with the property that $\lambda_{i} \in \lambda$ if and only if $2 n+1-\lambda_{i} \notin \lambda$, cf. [1, Section 9.3]. Given a non-degenerate skewsymmetric bilinear form on $\mathbb{C}^{2 n}$, fix an isotropic flag $F^{\bullet}$ in $\mathbb{C}^{2 n}$. The corresponding Schubert variety is

$$
Y_{\lambda}\left(F^{\bullet}\right)=\left\{E \in \operatorname{LG}(n, 2 n) \mid \operatorname{dim}\left(E \cap F^{\lambda_{k}}\right) \geq k, 1 \leq k \leq n\right\} .
$$

Define $\tilde{\lambda}=\left(\nu_{\mathrm{p}}^{c_{\mathrm{p}}}, \ldots, \nu_{0}^{c_{0}}\right)$ as in Section 2.2. Corollary 4.3, [19, Theorem 6.1], Remark A.7 and Lemma A.19 yield the following.

Theorem 2.3 (Schur rigidity in Lagrangian Grassmannians). The Schubert class $\xi_{\lambda} \in H_{\bullet}(\mathrm{LG}(n, 2 n), \mathbb{Z})$ is Schur rigid if and only if the 
following conditions hold:

(a) if $\nu_{\mathrm{p}}>0$, then $2 \leq c_{s}$, for all $1 \leq s \leq \mathrm{p}$;

(b) if $\nu_{\mathrm{p}}=0$, then $2 \leq c_{s}$, for all $0 \leq s \leq \mathrm{p}-1$.

See Table 1 (p. 11) for a list of Schur rigid Schubert classes in LG(5,10).

\subsection{Spinor varieties}

It is well-known that the Schubert varieties of $\mathcal{S}_{n}=D_{n} / P_{n}$ are indexed by partitions $1 \leq \lambda_{1}<\lambda_{2}<\cdots<\lambda_{n} \leq 2 n$ with the properties that $\lambda_{i} \in \lambda$ if and only if $2 n+1-\lambda_{i} \notin \lambda$, and \#\{i|$\left.\lambda_{i}>n\right\}$ is even, cf. [1, Section 9.3]. Given a non-degenerate symmetric bilinear form on $\mathbb{C}^{2 n}$, fix an isotropic flag $F^{\bullet}$ in $\mathbb{C}^{2 n}$. The corresponding Schubert variety is given by

$$
Y_{\lambda}\left(F^{\bullet}\right)=\left\{E \in \mathcal{S}_{n} \mid \operatorname{dim}\left(E \cap F^{\lambda_{k}}\right) \geq k, 1 \leq k \leq n\right\} .
$$

Decompose $\lambda=\hat{\mu}_{\mathrm{p}} \cdots \hat{\mu}_{1} \hat{\mu}_{0}$ into blocks $\hat{\mu}_{s}$ of "consecutive" integers, with the convention that the integers $n-1, n+1$ are considered consecutive and are placed in the same $\hat{\mu}_{s}$-block; likewise, the integers $n, n+2$ are consecutive. For example, if $n=5$, then $\lambda=(2,3,4,6,10)$ has block decomposition $\hat{\mu}_{1} \hat{\mu}_{0}=(2,3,4,6)(10)$; likewise, $\lambda=(1,2,5,7,8)$ has block decomposition $\hat{\mu}_{1} \hat{\mu}_{0}=(1,2)(5,7,8)$.

Let $\hat{c}_{s}=\left|\hat{\mu}_{s}\right|$ be the number of terms in the $s$-th block, and define

$$
r=r(\lambda)= \begin{cases}\lfloor p / 2\rfloor & \text { if } \lambda_{1}=1 \\ \lceil\mathrm{p} / 2\rceil & \text { if } \lambda_{1}>1\end{cases}
$$

Then $\hat{\mu}_{\mathrm{r}}$ is the block that has non-empty intersection with $\{n, n+1\}$. Corollary 4.3, [19, Theorem 6.1] and Lemma A.27 yield the following.

Theorem 2.4 (Schur rigidity in Spinor varieties). The Schubert class $\xi_{\lambda} \in H_{\bullet}\left(\mathcal{S}_{n}, \mathbb{Z}\right)$ is Schur rigid if and only if one of the following conditions hold:

(a) $\lambda_{1}>1,2 \leq \hat{c}_{s}$ for all $1 \leq s \leq \mathrm{p}$, and $3 \leq \hat{c}_{\mathrm{r}}$;

(b) $\lambda_{1}=1,2 \leq \hat{c}_{s}$ for all $0 \leq s \leq \mathrm{p}-1$, and $3 \leq \hat{c}_{\mathrm{r}}$.

See Table 2 (p. 12) for a list of Schur rigid Schubert classes in $\mathcal{S}_{6}$.

There exists an inclusion-preserving bijection between the set $\left\{Y_{\lambda^{\prime}} \subset\right.$ $\mathrm{LG}(n, 2 n)\}$ of Schubert varieties in the Lagrangian Grassmannian and the 
set $\left\{Y_{\lambda} \subset \mathcal{S}_{n+1}\right\}$ of Schubert varieties in the Spinor variety. Given a partition $\lambda=\left(\lambda_{1}, \ldots, \lambda_{n+1}\right)$ indexing a Schubert variety in $\mathcal{S}_{n+1}$, the corresponding Schubert variety in the Lagrangian Grassmannian is indexed by $\lambda^{\prime}=\left(\lambda_{1}^{\prime}, \ldots, \lambda_{n}^{\prime}\right)$, where

$$
\lambda^{\prime}= \begin{cases}\lambda_{j}^{\prime}=\lambda_{j}, & \text { if } \lambda_{j}<n+1 \\ \lambda_{j}^{\prime}=\lambda_{j}-2, & \text { if } \lambda_{j}>n+2\end{cases}
$$

Basically, $\lambda^{\prime}$ is obtained from $\lambda$ as follows: $\lambda$ contains precisely one of $\{n+1, n+2\}$; remove that integer, and shift those above down by two.

Theorems 2.3 and 2.4 yield the following.

Corollary 2.5. The Schubert class $\xi_{\lambda^{\prime}} \in H_{\bullet}(\mathrm{LG}(n, 2 n), \mathbb{Z})$ is Schur rigid if and only if the corresponding Schubert class $\xi_{\lambda} \in H_{\bullet}\left(\mathcal{S}_{n+1}, \mathbb{Z}\right)$ is Schur rigid.

The corollary is illustrated by a comparison of Tables 1 and 2 .

\section{Review}

\subsection{Notation and background}

This is a continuation of [19]. With the exception noted in Remark A.1, I will use the notation of that paper. To streamline the presentation, I will assume that the reader has reviewed the discussion of rational homogeneous varieties, their Schubert subvarieties, grading elements and Hasse posets

Table 1: Schubert varieties of $\operatorname{LG}(5,10)$, Schur rigid classes indicated by $*$.

\begin{tabular}{lc|cc|cc}
\hline$\lambda$ & $\mathrm{a}: \mathrm{J}$ & $\lambda$ & $\mathrm{a}: \mathrm{J}$ & $\lambda$ & $\mathrm{a}: \mathrm{J}$ \\
\hline$*(1,2,3,4,5)$ & & $(1,2,3,4,6)$ & $0: 4$ & $(1,2,3,5,7)$ & $1: 3,4$ \\
$(1,2,4,5,8)$ & $1: 2,4$ & $*(1,2,3,6,7)$ & $0: 3$ & $(1,3,4,5,9)$ & $1: 1,4$ \\
$(1,2,4,6,8)$ & $2: 2,3,4$ & $*(2,3,4,5,10)$ & $1: 4$ & $(1,3,4,6,9)$ & $2: 1,3,4$ \\
$(1,2,5,7,8)$ & $1: 2,3$ & $(2,3,4,6,10)$ & $2: 3,4$ & $(1,3,5,7,9)$ & $3: 1,2,3,4$ \\
$*(1,2,6,7,8)$ & $0: 2$ & $(2,3,5,7,10)$ & $3: 2,3,4$ & $*(1,4,5,8,9)$ & $1: 1,3$ \\
$(1,3,6,7,9)$ & $2: 1,2,4$ & $(2,4,5,8,10)$ & $3: 1,3,4$ & $*(2,3,6,7,10)$ & $2: 2,4$ \\
$(1,4,6,8,9)$ & $2: 1,2,3$ & $*(3,4,5,9,10)$ & $1: 3$ & $(2,4,6,8,10)$ & $4: 1,2,3,4$ \\
$(1,5,7,8,9)$ & $1: 1,2$ & $(3,4,6,9,10)$ & $2: 2,3$ & $(2,5,7,8,10)$ & $3: 1,2,4$ \\
$*(1,6,7,8,9)$ & $0: 1$ & $(3,5,7,9,10)$ & $3: 1,2,3$ & $(2,6,7,8,10)$ & $2: 1,4$ \\
$*(4,5,8,9,10)$ & $1: 2$ & $(3,6,7,9,10)$ & $2: 1,3$ & $(4,6,8,9,10)$ & $2: 1,2$ \\
$(5,7,8,9,10)$ & $1: 1$ & $*(6,7,8,9,10)$ & & & \\
\hline
\end{tabular}


Table 2: Schubert varieties of $\mathcal{S}_{6}$, Schur rigid classes indicated by $*$.

\begin{tabular}{lc|cccc}
\hline$\lambda$ & $\mathrm{a}: \mathrm{J}$ & $\mathrm{r}$ & $\lambda$ & $\mathrm{a}: \mathrm{J}$ & $\mathrm{r}$ \\
\hline$*(1,2,3,4,5,6)$ & & & $(1,2,3,4,7,8)$ & $0: 4$ & 0 \\
$(1,2,3,5,7,9)$ & $0: 3,5$ & 1 & $(1,2,4,5,7,10)$ & $0: 2,5$ & 1 \\
$*(1,2,3,6,8,9)$ & $0: 3$ & 0 & $(1,3,4,5,7,11)$ & $0: 1,5$ & 1 \\
$(1,2,4,6,8,10)$ & $1: 2,3,5$ & 1 & $*(2,3,4,5,7,12)$ & $0: 5$ & 1 \\
$(1,3,4,6,8,11)$ & $1: 1,3,5$ & 1 & $(1,2,5,6,9,10)$ & $1: 2,4$ & 1 \\
$(2,3,4,6,8,12)$ & $1: 3,5$ & 1 & $(1,3,5,6,9,11)$ & $2: 1,2,4,5$ & 2 \\
$*(1,2,7,8,9,10)$ & $0: 2$ & 0 & $(2,3,5,6,9,12)$ & $2: 2,4,5$ & 2 \\
$*(1,4,5,6,10,11)$ & $1: 1,4$ & 1 & $(1,3,7,8,9,11)$ & $1: 1,2,5$ & 1 \\
$(2,4,5,6,10,12)$ & $2: 1,4,5$ & 2 & $*(2,3,7,8,9,12)$ & $1: 2,5$ & 1 \\
$(1,4,7,8,10,11)$ & $2: 1,2,4$ & 1 & $*(3,4,5,6,11,12)$ & $1: 4$ & 1 \\
$(2,4,7,8,10,12)$ & $3: 1,2,4,5$ & 2 & $(1,5,7,9,10,11)$ & $1: 1,3$ & 1 \\
$(3,4,7,8,11,12)$ & $2: 2,4$ & 1 & $(2,5,7,9,10,12)$ & $2: 1,3,5$ & 2 \\
$*(1,6,8,9,10,11)$ & $0: 1$ & 0 & $(3,5,7,9,11,12)$ & $3: 1,3,4$ & 2 \\
$(2,6,8,9,10,12)$ & $1: 1,5$ & 1 & $*(4,5,7,10,11,12)$ & $1: 3$ & 1 \\
$(3,6,8,9,11,12)$ & $2: 1,4$ & 1 & $(4,6,8,10,11,12)$ & $2: 1,3$ & 1 \\
$(5,6,9,10,11,12)$ & $1: 2$ & 1 & $*(7,8,9,10,11,12)$ & & \\
\hline
\end{tabular}

in [19, Sections 2.1 to 2.4 and 3.1]. Briefly, $G$ is a connected complex simple Lie group. A choice of Cartan and Borel subgroups $H \subset B$ has been fixed, $P \supset B$ is a maximal parabolic subgroup associated with a cominuscule root, and $X=G / P$ is the corresponding cominuscule variety. The associated Lie algebras are denoted $\mathfrak{h} \subset \mathfrak{b} \subset \mathfrak{p} \subset \mathfrak{g}$. Let $W$ denote the Weyl group of $\mathfrak{g}$, and $W_{\mathfrak{p}}$ the Weyl group of the reductive component in the Levi decomposition of $\mathfrak{p}$. The Hasse poset $W^{\mathfrak{p}}$ is the set of minimal length representatives of the coset space $W_{\mathfrak{p}} \backslash W$, and indexes the Schubert classes. Let

$$
o=P / P \in X=G / P .
$$

Given $w \in W^{\mathfrak{p}}$, the Zariski closure

$$
Y_{w}=\overline{B w^{-1} \cdot o}
$$

is a Schubert variety. Any $G$-translate of the Schubert variety $Y_{w}$ will be referred to as a Schubert variety of type $w$. Let $\xi_{w}=\left[Y_{w}\right] \in H_{2|w|}(X, \mathbb{Z})$ denote the corresponding Schubert class. 
Let $\left\{Z_{1}, \ldots, Z_{r}\right\}$ be the basis of $\mathfrak{h}$ dual to the simple roots $\left\{\alpha_{1}, \ldots, \alpha_{r}\right\}$. Let $\alpha_{\mathfrak{i}}$ be the simple root associated with the cominuscule $\mathfrak{p}$. Then $\mathfrak{g}$ decomposes into $Z_{i}$-eigenspaces

$$
\mathfrak{g}=\mathfrak{g}_{1} \oplus \mathfrak{g}_{0} \oplus \mathfrak{g}_{-1}, \quad \text { where } \mathfrak{g}_{k}:=\left\{A \in \mathfrak{g} \mid\left[Z_{\mathfrak{i}}, A\right]=k A\right\}
$$

we call this eigenspace decomposition the $Z_{i}$-graded decomposition of the Lie algebra $\mathfrak{g}$. Moreover,

$$
\mathfrak{p}=\mathfrak{g}_{1} \oplus \mathfrak{g}_{0},
$$

and $\mathfrak{g}_{0}$ is the reductive component of the parabolic subalgebra $\mathfrak{p}$.

Remark 3.3. By the Jacobi identity, $\left[\mathfrak{g}_{k}, \mathfrak{g}_{\ell}\right] \subset \mathfrak{g}_{k+\ell}$. In particular, the subalgebras $\mathfrak{g}_{ \pm 1} \subset \mathfrak{g}$ are abelian, $\left[\mathfrak{g}_{1}, \mathfrak{g}_{1}\right]=\{0\}=\left[\mathfrak{g}_{-1}, \mathfrak{g}_{-1}\right]$.

Let $\Delta$ denote the set of roots of $\mathfrak{g}$. Given $\alpha \in \Delta$, let $\mathfrak{g}_{\alpha} \subset \mathfrak{g}$ denote the corresponding root space. Given any subset $\mathfrak{s} \subset \mathfrak{g}$, let

$$
\Delta(\mathfrak{s})=\left\{\alpha \in \Delta \mid \mathfrak{g}_{\alpha} \subset \mathfrak{s}\right\}
$$

Given a subset $U$ of a vector space, let $\langle U\rangle$ denote the linear span.

\subsection{The Schur differential system}

There is a natural $\mathfrak{g}_{0}$-module identification

$$
T_{o} X \simeq \mathfrak{g}_{-1}
$$

Kostant [16, Corollary 8.2] showed that the $\ell$-th exterior power decomposes as

$$
\bigwedge^{\ell} T_{o} X=\bigoplus_{\substack{w \in W^{\mathfrak{p}} \\|w|=\ell}} \mathbf{I}_{w}
$$

into irreducible $\mathfrak{g}_{0}$-modules. The highest weight line $\mathfrak{n}_{w} \in \mathbb{P} \mathbf{I}_{w}$ is defined by

$$
\Delta(w)=w \Delta^{-} \cap \Delta^{+} \quad \text { and } \quad \mathfrak{n}_{w}=\bigoplus_{\alpha \in \Delta(w)} \mathfrak{g}_{-\alpha}
$$

Remark 3.5. One important consequence of Remark 3.3 is that given a set $\Phi \subset \Delta\left(\mathfrak{g}_{1}\right)$, there exists $w \in W^{\mathfrak{p}}$ such that $\Delta(w)=\Phi$ if and only if $\Delta^{+} \backslash \Phi$ is closed. For details see [19, Section 2.3]. 
Consider the set of tangent $\ell$-planes $\operatorname{Gr}\left(\ell, T_{o} X\right)$ as a subvariety of $\mathbb{P}\left(\bigwedge^{\ell} T_{o} X\right)$ via the Plücker embedding, and set $R_{w}=\operatorname{Gr}\left(|w|, T_{o} X\right) \cap \mathbb{P} \mathbf{I}_{w}$. The Schur system is the homogeneous bundle $\mathcal{R}_{w} \subset \operatorname{Gr}(|w|, T X)$ with fiber $R_{w}$ over $o \in X$. Given a complex submanifold $M \subset X$ of dimension $|w|$, let $\mathcal{M} \subset \operatorname{Gr}(|w|, T X)$ denote the canonical lift. An integral manifold $M$ of the Schur system is a complex submanifold $M \subset X$ with the property that $\mathcal{M} \subset \mathcal{R}_{w}$. The Schur system is rigid if every connected integral manifold is contained in a Schubert variety of type $w$. A connected integral manifold that is not contained in any Schubert variety of type $w$ we call non-trivial. When there exist non-trivial integrals we say the Schur system is flexible.

An integral variety of the Schur system is a subvariety $Y \subset X$ with the property that the set of smooth points $Y^{0}$ is an integral manifold of the Schur system. The Schubert class $\xi_{w}$ (or the Schubert variety $Y_{w}$ ) is Schur rigid if every irreducible integral variety of the Schur system is a Schubert variety of type $w$. An irreducible integral variety that is not Schubert variety of type $w$ is non-trivial. When there exist non-trivial integral varieties we say the Schubert class $\xi_{w}$ (or the Schubert variety $Y_{w}$ ) is Schur flexible.

Theorem 3.6 $[\mathbf{3}, \mathbf{2 1}]$. The homology class $[Y] \subset H_{2|w|}(X, \mathbb{Z})$ represented by a variety $Y \subset X$ is an integer multiple of the Schubert class $\xi_{w}$ if and only if $Y$ is an integral of the Schur system $\mathcal{R}_{w}$.

Remark 3.7. A priori, it may happen that $\mathcal{R}_{w}$ admits non-trivial integral manifolds (a differential-geometric property), but no non-trivial integral varieties (an algebraic-geometric property). That is, it may be the case that $\mathcal{R}_{w}$ is flexible, while $\xi_{w}$ is Schur rigid. However, we will see a posteriori (Corollary 4.3) that $\mathcal{R}_{w}$ is flexible if and only if $\xi_{w}$ is Schur flexible.

For more on the Schur system, see [19, Section 7] and references therein.

\subsection{The Schubert differential system}

Recall the $Z_{\mathfrak{i}}$-graded decomposition $(3.1)$ of $\mathfrak{g}$, and let

$$
G_{0}:=\left\{g \in G \mid \operatorname{Ad}_{g}\left(\mathfrak{g}_{j}\right) \subset \mathfrak{g}_{j}\right\}
$$

Then $G_{0}$ is a closed subgroup of $G$ with Lie algebra $\mathfrak{g}_{0}$. Associated to the Schur system is the more restrictive Schubert system $\mathcal{B}_{w}$ defined by the $G_{0^{-}}$ orbit $B_{w} \subset \operatorname{Gr}\left(|w|, T_{o} X\right)$ of the $\mathfrak{g}_{0}$-highest weight line $\mathfrak{n}_{w} \in R_{w}$. In particular, $B_{w} \subset R_{w}$. The corresponding homogeneous bundle $\mathcal{B}_{w} \subset \operatorname{Gr}(|w|, T X)$ is precisely the set of $|w|$-planes tangent to (a smooth point of) a Schubert 
variety of type $w$. The notions of integrals, rigidity and flexibility for the Schubert system are analogous to those for the Schur system (Section 3.2). Theorem 3.6 and $\mathcal{B}_{w} \subset \mathcal{R}_{w}$ yield

Corollary 3.8. The homology class $[Y] \subset H_{2|w|}(X, \mathbb{Z})$ represented by an integral variety $Y \subset X$ of the Schubert system $\mathcal{B}_{w}$ is an integer multiple of the Schubert class $\xi_{w}$.

For more on the Schubert system see [19, Section 4] and references therein. The following is due to Bryant (in the case that $X$ is a Grassmannian) and Hong (in general).

Proposition 3.9. The Schur system $\mathcal{R}_{w}$ is rigid if and only if $B_{w}=R_{w}$ and the Schubert system $\mathcal{B}_{w}$ is rigid.

Remark 3.10. (a) $A$ posteriori the condition that $R_{w}=B_{w}$ may be dropped; it is a consequence of Theorem 4.1 and Corollary 4.3 that $\mathcal{B}_{w}$ is rigid if and only if $\mathcal{R}_{w}$ is rigid.

(b) Note that $R_{w}$ is the intersection of $\operatorname{Gr}\left(|w|, T_{o} X\right)$ with the (projective) linear span of $B_{w}$ in $\mathbb{P}\left(\bigwedge^{|w|} T_{o} X\right)$. In general, the containment $B_{w} \subset R_{w}$ is strict.

The Schubert system lifts to a linear Pfaffian EDS defined on a frame bundle over $X$ [19, Section 4.4]. The lifted system has the advantage that obstructions to rigidity may be identified using Lie algebra cohomology; cf. [19, Sections 4 and 5]. A complete list of the Schubert systems $\mathcal{B}_{w}$ for which there exist no cohomological obstructions to rigidity is given by [19, Theorem 6.1]: these classes are Schubert rigid. It is then shown in [19, Theorem 8.1] that $R_{w}=B_{w}$ for each of these rigid systems. In particular, the corresponding Schur system $\mathcal{R}_{w}$ is rigid.

The goal of this paper is to prove that these cohomological obstructions are genuine obstructions to the Schubert rigidity of $\xi_{w}$ : that is, given cohomological obstructions there exist non-trivial, algebraic integrals $Y$ of the Schubert system (Theorem 4.1). As an integral of $\mathcal{B}_{w}$, the variety $Y$ is necessarily an integral of the Schur system $\mathcal{R}_{w}$. In particular, $[Y]=r \xi_{w}$ for some integer $r>0$.

\subsection{The characterization of Schubert varieties}

This section is a brief review of the characterization of Schubert classes $\xi_{w}$ by an integer $\mathrm{a}(w) \geq 0$ and a marking $\mathrm{J}(w)$ of the Dynkin diagram. 
(The marking is equivalent to a choice of simple roots.) For more detail see [19, Section 3.2]. Recall (3.4), and let

$$
N_{w}=\exp \left(\mathfrak{n}_{w}\right)
$$

Then

$$
X_{w}:=\overline{N_{w} \cdot o}=w Y_{w}
$$

is a Schubert variety of type $w$. (See [19, Sections 2.3 and 2.4] for more detail.)

Let $1 \in W^{\mathfrak{p}}$ be the identity, and let $w_{0} \in W^{\mathfrak{p}}$ be the longest element. The $\mathfrak{g}_{0}$-module $\mathbf{I}_{w}$ is trivial if and only if $w \in\left\{1, w_{0}\right\}$. (The associated Schubert varieties are $X_{1}=o$ and $X_{w_{0}}=X$.) Assume $w \in W^{\mathfrak{p}} \backslash\left\{1, w_{0}\right\}$. Let $\mathfrak{q}_{w} \subset \mathfrak{g}_{0}$ be the stabilizer of the highest weight line $\mathfrak{n}_{w} \in \mathbb{P}\left(\wedge^{|w|} T_{o} X\right)$. Then there is a subset $\mathrm{J}(w) \subset\{1, \ldots, r\} \backslash\{i\}$ with the property that the Lie algebra $\mathfrak{q}_{w}$ is given by $\mathfrak{q}_{w}=\mathfrak{g}_{0, \geq 0}$, where

$$
\mathfrak{g}_{k, \ell}:=\left\{A \in \mathfrak{g}_{k} \mid\left[Z_{w}, A\right]=\ell A\right\} \quad \text { and } \quad Z_{w}=\sum_{j \in \mathrm{J}(w)} Z_{\mathrm{j}}
$$

We call $\mathfrak{g}=\oplus \mathfrak{g}_{k, \ell}$ the $\left(Z_{\mathfrak{i}}, Z_{w}\right)$-bigraded decomposition of $\mathfrak{g}$; it is the decomposition of $\mathfrak{g}$ into $\left(Z_{\mathfrak{i}}, Z_{w}\right)$-eigenspaces. The following is [19, Proposition $3.9]$.

Proposition 3.13 [19]. Let $w \in W^{\mathfrak{p}} \backslash\left\{1, w_{0}\right\}$. There exists an integer $\mathrm{a}=$ $\mathrm{a}(w) \geq 0$ such that $\Delta(w)=\left\{\alpha \in \Delta\left(\mathfrak{g}_{1}\right) \mid \alpha\left(Z_{w}\right) \leq \mathrm{a}\right\}$. Equivalently,

$$
\mathfrak{n}_{w}=\mathfrak{g}_{-1,0} \oplus \cdots \oplus \mathfrak{g}_{-1,-a} \text {. }
$$

Remark 3.15. (a) Since $\xi_{w}=\left[X_{w}\right]$, and $X_{w}$ is determined by $\Delta(w)$, the pair a $(w), \mathrm{J}(w)$ characterizes $\xi_{w}$, where $w \in W^{\mathfrak{p}} \backslash\left\{1, w_{0}\right\}$.

(b) By [19, Proposition 3.19], the Schubert variety $X_{w}$ is smooth if and only if $\mathrm{a}(w)=0$. For more on the relationship between the integer $\mathrm{a}(w)$ and $\operatorname{Sing}\left(X_{w}\right)$, see [18].

(c) A tableau-esque analog of Proposition 3.13 is given by Thomas and Yong in [20, Proposition 2.1].

A complete list of the $\mathrm{a}(w), \mathrm{J}(w)$ that occur is given by [19, Corollary 3.17]. For each of the classical, cominuscule varieties, the relationship between $\mathrm{a}(w), \mathrm{J}(w)$ and the familiar geometric descriptions of $Y_{w}$ is reviewed in the appendix. 


\subsection{Divisors}

In this section, we establish a lemma that will be used in the proof of Theorem 4.1. Recall the $\left(Z_{\mathfrak{i}}, Z_{w}\right)$-bigraded decomposition (3.12) of $\mathfrak{g}$, and note that $\mathfrak{g}_{0,0}$ is a reductive subalgebra of $\mathfrak{g}$.

Definition 3.16. Given a representation $U$ of $\mathfrak{g}_{0,0}$, let $\Pi(U)$ denote the set of highest weights.

Given $\alpha \in \Delta^{+}$, let $r_{\alpha} \in W$ denote the corresponding reflection.

Lemma 3.17. Let $\alpha \in \Delta(w)$. Then $\Delta(w)=\Delta\left(w^{\prime}\right) \sqcup\{\alpha\}$ for some $w^{\prime} \in$ $W^{\mathfrak{p}}$ if and only if $\alpha \in \Pi\left(\mathfrak{g}_{1, \mathrm{a}}\right)$. In this case $w^{\prime}=r_{\alpha} w$.

Proof. By Remark 3.5, there exists $w^{\prime} \in W^{\mathfrak{p}}$ such that $\Delta(w) \backslash\{\alpha\}=\Delta\left(w^{\prime}\right)$ if and only if $\Phi=\{\alpha\} \cup\left(\Delta^{+} \backslash \Delta(w)\right)$ is closed. So, it suffices to show that $\alpha \in \Pi\left(\mathfrak{g}_{1, \mathrm{a}}\right)$ if and only if $\Phi$ is closed.

Assume $\alpha \in \Pi\left(\mathfrak{g}_{1, \mathrm{a}}\right)$. Let $\mu, \nu \in \Phi$, and suppose that $\mu+\nu \in \Delta$. To see that $\Phi$ is closed, we must show that $\mu+\nu \in \Phi$. First, note that either $\mu \neq \alpha$ or $\nu \neq \alpha$, as $2 \alpha \notin \Delta$. Second, if both $\mu, \nu \in \Delta^{+} \backslash \Delta(w)$, then $\mu+\nu \in$ $\Delta^{+} \backslash \Delta(w) \subset \Phi$, by Remark 3.5. It remains to consider the case that $\mu=\alpha$ and $\nu \in \Delta^{+} \backslash \Delta(w)$. Remark 3.3, and the assumption that $\mu+\nu$ is a root, force $\nu \in \Delta^{+}\left(\mathfrak{g}_{0}\right)$. Note that $\nu\left(Z_{w}\right) \geq 0$. If $\nu\left(Z_{w}\right)=0$, then $\nu$ is a positive root of $\mathfrak{g}_{0,0}$, and the hypothesis that $\alpha$ is a highest $\mathfrak{g}_{0,0}$-weight implies $\mu+\nu \notin \Delta$. If $\nu\left(Z_{w}\right)>0$, then $(\mu+\nu)\left(Z_{w}\right)>$ a. By Proposition 3.13, we have $\Delta^{+} \backslash \Delta(w)=\Delta^{+}\left(\mathfrak{g}_{0}\right) \cup \Delta\left(\mathfrak{g}_{1,>\mathrm{a}}\right)$; in particular, $\mu+\nu \in \Delta^{+} \backslash \Delta(w) \subset \Phi$.

Conversely, suppose that $\Phi$ is closed. To see that $\alpha$ is a highest $\mathfrak{g}_{0,0^{-}}$ weight, it suffices to show that $\alpha+\beta \notin \Delta$ for all $\beta \in \Sigma\left(\mathfrak{g}_{0,0}\right)$. Note that $(\alpha+$ $\beta)\left(Z_{w}\right)=\mathrm{a}+0$. So, if $\alpha+\beta \in \Delta$, then $\alpha+\beta \in \Delta\left(\mathfrak{g}_{1, \mathrm{a}}\right)$. By Proposition 3.13, $\Delta\left(\mathfrak{g}_{1, \mathrm{a}}\right) \subset \Delta(w)$. Therefore, $\alpha+\beta \in \Delta^{+} \backslash \Phi$; since $\alpha, \beta \in \Phi$, this contradicts the assumption that $\Phi$ is closed.

It remains to show that $w^{\prime}=r_{\alpha} w$. This is [5, Proposition 3.2.15(3)].

\section{Flexibility}

In $\left[19\right.$, Section 4] we saw that a Lie algebra cohomology $H^{1}\left(\mathfrak{n}_{w}, \mathfrak{g}_{w}^{\perp}\right)$ group is naturally associated with the Schubert system $\mathcal{B}_{w}$. The cohomology group contains two distinguished subspaces $H_{1, \mathrm{a}-1}^{1}$ and $H_{2,2 \mathrm{a}-1}^{1}$. When the subspaces are trivial, the Schubert system is necessarily rigid, [19, Theorem 5.38]. The two subspaces are trivial precisely when two representation theoretic conditions, $\mathrm{H}_{1}=\mathrm{H}_{1}(w)$ and $\mathrm{H}_{2}=\mathrm{H}_{2}(w)$, are satisfied (Definition 4.6). 
When both conditions hold, we say that $\mathrm{H}_{+}$is satisfied. In summary: define

$$
\mathcal{O}_{w}=H_{1, \mathrm{a}-1}^{1} \oplus H_{2,2 \mathrm{a}-1}^{1} ;
$$

then

$$
\mathcal{O}_{w}=0 \text { if and only if } \mathbf{H}_{+} \text {holds. }
$$

Theorem 4.1. Let $X=G / P$ be a cominuscule variety, and fix $w \in$ $W^{\mathfrak{p}} \backslash\left\{1, w_{0}\right\}$. The Schubert system $\mathcal{B}_{w}$ is rigid if and only if $\mathcal{O}_{w}=0$. Moreover, when $\mathcal{O}_{w} \neq 0$, there exist non-trivial algebraic integrals; in particular, the Schubert class $\xi_{w}$ is Schubert rigid if and only if $\mathcal{O}_{w}=0$.

Remark 4.2 (Geometric interpretation). Recall (Section 3.3) that an irreducible variety $Y \subset X$ is an integral of the Schubert system $\mathcal{B}_{w}$ if and only if at each smooth point $y \in Y$ there exists a Schubert variety $Y^{\prime}=$ $Y^{\prime}(y)$ of type $w$, such that $y$ is also a smooth point of $Y^{\prime}$, and $T_{y} Y=T_{y} Y^{\prime}$. Equivalently, if $\mathcal{Y}$ denotes the canonical lift of the smooth locus $Y_{0} \subset Y$ to $\operatorname{Gr}(|w|, T X)$, then $\mathcal{Y}$ and $\mathcal{Y}^{\prime}$ intersect at $\tilde{y}=T_{y} Y \in \operatorname{Gr}(|w|, T X)$. If the condition $\mathrm{H}_{1}$ holds, then $\mathcal{Y}$ and $\mathcal{Y}^{\prime}$ are necessarily tangent at $\tilde{y}$; and when both conditions $\mathrm{H}_{1}$ and $\mathrm{H}_{2}$ hold, $\mathcal{Y}$ and $\mathcal{Y}^{\prime}$ agree to second-order at $\tilde{y}$.

Corollary 4.3. Let $w \in W^{\mathfrak{p}} \backslash\left\{1, w_{0}\right\}$. The Schur $\mathcal{R}_{w}$ system is rigid if and only if $\mathcal{O}_{w}=0$. Moreover, when $\mathcal{O}_{w} \neq 0$, there exist non-trivial algebraic integrals; in particular, the Schubert class $\xi_{w}$ is Schur rigid if and only if $\mathcal{O}_{w}=0$.

Proof. By [19, Theorem 8.1] if $\mathcal{O}_{w}=0$, then $B_{w}=R_{w}$. The corollary then follows from Proposition 3.9 and Theorem 4.1.

Remark 4.4. A complete list of the Schubert varieties (in an irreducible cominuscule $X$ ) satisfying $\mathrm{H}_{+}$is given by [19, Theorem 6.1].

Corollary 4.5 (Poincaré duality). A Schubert class $\xi_{w}$ is Schur rigid if and only if its Poincaré dual $\xi_{w^{*}}$ is Schur rigid.

Proof. Given $w \in W^{\mathfrak{p}}$, let $w^{*} \in W^{\mathfrak{p}}$ be the element associated to the Poincaré dual of $\xi_{w}$. By [19, Corollary 6.2], the condition $\mathrm{H}_{+}(w)$ holds if and only if $\mathrm{H}_{+}\left(w^{*}\right)$ holds. It follows from Theorem 4.1 (resp. Corollary 4.3) that the Schubert system $\mathcal{B}_{w}$ (resp. the Schur system $\mathcal{R}_{w}$ ) is rigid if and only if the system $\mathcal{B}_{w^{*}}\left(\right.$ resp. $\left.\mathcal{R}_{w^{*}}\right)$ is rigid. 
As given by [19, Definitions 5.27 and 5.36], we have

Definition 4.6. The condition $\mathrm{H}_{1}$ fails when there exist $\mathfrak{g}_{0,0}$-highest weights $-\beta \in \Delta\left(\mathfrak{g}_{0,-1}\right)$ and $\gamma \in \Delta\left(\mathfrak{g}_{1, \mathrm{a}}\right)$ such that

$$
\left[\mathfrak{g}_{-\beta}, \mathfrak{g}_{\gamma}\right] \stackrel{(\mathrm{i})}{=}\{0\} \neq\left[\mathfrak{g}_{\beta}, \mathfrak{g}_{\gamma}\right] \stackrel{(\mathrm{ii})}{=}\left[\mathfrak{g}_{\beta}, \mathfrak{g}_{1, \mathrm{a}}\right]
$$

The condition $\mathrm{H}_{2}$ fails when there exist $\mathfrak{g}_{0,0}$-highest weights $\varepsilon \in \Delta\left(\mathfrak{g}_{1, \mathrm{a}-1}\right)$ and $\gamma \in \Delta\left(\mathfrak{g}_{1, \mathrm{a}}\right)$ such that

$$
\{0\} \stackrel{(\mathrm{i})}{\neq}\left[\mathfrak{g}_{\varepsilon}, \mathfrak{g}_{-\gamma}\right] \stackrel{(\mathrm{ii})}{=}\left[\mathfrak{g}_{\varepsilon}, \mathfrak{g}_{-1,-\mathrm{a}}\right]
$$

When both $\mathrm{H}_{1}$ and $\mathrm{H}_{2}$ hold, we say that the condition $\mathrm{H}_{+}$is satisfied.

Remark 4.9. Note that $-\beta$ is a $\mathfrak{g}_{0,0}$-highest weight if and only if $\beta$ is a $\mathfrak{g}_{0,0}$-lowest weight. That is, $\beta \in \Delta\left(\mathfrak{g}_{0,1}\right)$ is a simple root.

Remark 4.10. Note that $\mathrm{H}_{2}$ is trivially satisfied when $\mathrm{a}=0$, as $\mathfrak{g}_{1, \mathrm{a}-1}=$ $\mathfrak{g}_{1,-1}=0$.

Example 4.11. In Example A.3, the $\mathfrak{g}_{0,0}$-lowest weights $\beta \in \Delta\left(\mathfrak{g}_{0,1}\right)$ are $\left\{\alpha_{2}, \alpha_{3}, \alpha_{7}, \alpha_{9}, \alpha_{12}\right\}$, and the $\mathfrak{g}_{0,0}$-highest weights are

$$
\begin{aligned}
\Pi\left(\mathfrak{g}_{1, \mathrm{a}}\right) & =\left\{\alpha_{1}+\cdots+\alpha_{6}, \alpha_{3}+\cdots+\alpha_{8}, \alpha_{4}+\cdots+\alpha_{11}\right\} \\
\Pi\left(\mathfrak{g}_{1, \mathrm{a}-1}\right) & =\left\{\alpha_{3}+\cdots+\alpha_{6}, \alpha_{4}+\cdots+\alpha_{8}\right\} .
\end{aligned}
$$

The condition $\mathrm{H}_{1}$ fails for $\beta=\alpha_{9}$ and $\gamma=\alpha_{3}+\cdots+\alpha_{8}$. The condition $\mathrm{H}_{2}$ is satisfied.

Example 4.12. In Example A.17, the $\mathfrak{g}_{0,0}$-lowest weights $\beta \in \Delta\left(\mathfrak{g}_{0,1}\right)$ are $\left\{\alpha_{1}, \alpha_{2}, \alpha_{4}\right\}$, and the $\mathfrak{g}_{0,0}$-highest weights are

$$
\begin{aligned}
\Pi\left(\mathfrak{g}_{1, \mathrm{a}}\right) & =\left\{\alpha_{1}+\cdots+\alpha_{5}, \alpha_{2}+2 \alpha_{3}+2 \alpha_{4}+\alpha_{5}\right\}, \\
\Pi\left(\mathfrak{g}_{1, \mathrm{a}-1}\right) & =\left\{\alpha_{2}+\cdots+\alpha_{5}, 2 \alpha_{3}+2 \alpha_{4}+\alpha_{5}\right\} .
\end{aligned}
$$

The condition $\mathrm{H}_{1}$ fails for $\beta=\alpha_{4}$ and $\gamma=\alpha_{1}+\cdots+\alpha_{5}$. The condition $\mathrm{H}_{2}$ fails for $\varepsilon=2 \alpha_{3}+2 \alpha_{4}+\alpha_{5}$ and $\gamma=\varepsilon+\alpha_{2}$. 


\subsection{Outline of the proof of Theorem 4.1}

By $\left[19\right.$, Theorem 5.38], the Schubert system $\mathcal{B}_{w}$ is rigid when $\mathbf{H}_{+}$is satisfied. So to prove Theorem 4.1 if suffices to show that there exist non-trivial integrals $Y$ of the Schubert system when $\mathrm{H}_{+}$fails. This is done in Sections 4.2 and 4.3. We will see that there exists a Schubert divisor $X_{w^{\prime}} \subset X_{w}$ and a 1-parameter subgroup $A \subset G$ with the property that $Y=\overline{A \cdot X_{w^{\prime}}}$ is a nontrivial integral variety of the Schubert system $\mathcal{B}_{w}$.

To see that the varieties $Y$ constructed in Sections 4.2 and 4.3 are nontrivial integrals of the Schubert system we must review some of the results of [19]. Define

$$
\mathfrak{n}_{w}^{\perp}=\bigoplus_{\alpha \in \Delta\left(\mathfrak{g}_{1}\right) \backslash \Delta(w)} \mathfrak{g}_{-\alpha}=\bigoplus_{b>a} \mathfrak{g}_{-1,-b},
$$

so that $\mathfrak{g}_{-1}=\mathfrak{n}_{w} \oplus \mathfrak{n}_{w}^{\perp}$, and let

$$
\mathfrak{g}_{w}^{\perp}=\mathfrak{n}_{w}^{\perp} \oplus \mathfrak{g}_{0,-} \oplus \mathfrak{g}_{1,<\mathrm{a}} .
$$

Each cohomology class $[\nu] \in H^{1}\left(\mathfrak{n}_{w}, \mathfrak{g}_{w}^{\perp}\right)$ admits a unique harmonic representative $\nu \in \mathcal{H}^{1} \subset \mathfrak{g}_{w}^{\perp} \otimes \mathfrak{n}_{w}^{*}$ by [19, Proposition 5.10]. The grading element $Z_{\mathrm{i}}$ induces a graded decomposition $\mathcal{H}^{1}=\mathcal{H}_{0}^{1} \oplus \mathcal{H}_{1}^{1} \oplus \mathcal{H}_{2}^{1}$ with $\mathcal{H}_{0} \subset \mathfrak{n}_{w}^{\perp} \otimes \mathfrak{n}_{w}^{*}$, $\mathcal{H}_{1}^{1} \subset \mathfrak{g}_{0,-} \otimes \mathfrak{n}_{w}^{*}$ and $\mathcal{H}_{2}^{1} \subset \mathfrak{g}_{1,<a} \otimes \mathfrak{n}_{w}^{*}$.

Let $\vartheta$ denote the $\mathfrak{g}$-valued, left-invariant Maurer-Cartan form on $G$. Set $\mathfrak{g}_{w}=\mathfrak{n}_{w} \oplus \mathfrak{g}_{0, \geq 0} \oplus \mathfrak{g}_{1, \geq \mathbf{a}}$, so that $\mathfrak{g}=\mathfrak{g}_{w} \oplus \mathfrak{g}_{w}^{\perp}$. Let $\vartheta_{k, \ell}$ denote the $\mathfrak{g}_{k, \ell^{-}}$ valued component of the Maurer-Cartan form, with respect to the direct sum decomposition $\mathfrak{g}=\oplus \mathfrak{g}_{k, \ell}$ of (3.12). Similarly, let $\vartheta_{\mathfrak{g}_{w}}$ and $\vartheta_{\mathfrak{n}_{w}^{\perp}}$ respectively denote the $\mathfrak{g}_{w}$-and $\mathfrak{n}_{w}^{\perp}$-valued components of $\vartheta$, with respect to the direct sum decompositions $\mathfrak{g}=\left(\mathfrak{n}_{w} \oplus \mathfrak{n}_{w}^{\perp}\right) \oplus \mathfrak{g}_{\geq 0}=\mathfrak{g}_{w} \oplus \mathfrak{g}_{w}^{\perp}$. The following is an amalgam of [19, Lemma 4.9 and Corollary 5.12].

Lemma 4.13. There is a bijective correspondence between submanifolds $U \subset G$ such that

(i) $\vartheta_{\mathfrak{g}_{w}}: T_{g} U \rightarrow \mathfrak{g}_{w}$ is a linear isomorphism for all $g \in U$,

(ii) $\vartheta_{\mathfrak{n}_{w}^{\perp}}=0$ vanishes on $U$,

(iii) there exists a smooth map $\lambda: U \rightarrow \mathcal{H}_{1}^{1}$ such that $\vartheta_{0,-}=\lambda\left(\vartheta_{\mathfrak{n}_{w}}\right)$ on $U$,

and integral manifolds $U \cdot o \subset X$ of the Schubert system. Additionally, there exists a smooth function $\mu: U \rightarrow \mathfrak{g}_{1,<\mathrm{a}} \otimes \mathfrak{n}_{w}^{*}$ such that $\vartheta_{1,<\mathrm{a}}=\mu\left(\vartheta_{\mathfrak{n}_{w}}\right)$ on $U$. If $\lambda$ is identically zero, then $\mu$ takes values in $\mathcal{H}_{2}^{1}$. 
The submanifold $U$ of Lemma 4.13 is the subbundle $\mathcal{F}^{0}$ of $[19$, Corollary 5.12]. It is a consequence of the arguments of [19, Section 4.7] that the subbundle $\mathcal{F}^{0}$ is unique. Then [19, Proposition 4.13] yields

Lemma 4.14. The integral manifold $U \cdot o \subset X$ is a Schubert variety of type $w$ if and only if both $\lambda$ and $\mu$ vanish identically on $U$.

The integrals $Y=\overline{A \cdot X_{w^{\prime}}}$ constructed in Sections 4.2 and 4.3 will be of the form $Y=\overline{U \cdot o}$, where $U$ satisfies the hypothesis of Lemma 4.15.

Lemma 4.15. Let $U \subset G$ be a submanifold such that

(i) $\vartheta_{\mathfrak{g}_{w}}: T_{g} U \rightarrow \mathfrak{n}_{w}$ is a linear isomorphism for all $g \in U$,

(ii) $\vartheta_{\mathfrak{n}_{w}^{\perp}}$ vanishes on $U$.

Then $U \cdot o \subset X$ is a non-trivial integral manifold of the Schubert system if either

(a) there exists a smooth map $\lambda: U \rightarrow \mathcal{H}_{1}^{1}$, that is not identically zero, such that $\vartheta_{0,-}=\lambda\left(\vartheta_{\mathfrak{n}_{w}}\right)$ on $U$; or

(b) $\vartheta_{0,-}$ vanishes on $U$, and there exists a smooth map $\mu: U \rightarrow \mathcal{H}_{2}^{1}$, that is not identically zero, such that $\vartheta_{1,<\mathrm{a}}=\mu\left(\vartheta_{\mathfrak{n}_{w}}\right)$.

Proof. Let $G^{\prime} \subset G$ be the connected Lie group with Lie algebra $\mathfrak{g}_{0, \geq 0} \oplus \mathfrak{g}_{1, \geq \mathrm{a}}$. Let $U \subset G$ be a submanifold satisfying (i) and (ii) of Lemma 4.15. Then $U^{\prime}=U G^{\prime} \subset G$ is a submanifold satisfying (i) and (ii) of Lemma 4.13.

Suppose $U$ satisfies Lemma 4.15(a). Then $U^{\prime}$ satisfies Lemma 4.13(iii). Lemma 4.14 implies that $U \cdot o=U^{\prime} \cdot o \subset X$ is a non-trivial integral of the Schubert system.

Suppose $U$ satisfies Lemma $4.15(\mathrm{~b})$. The $\vartheta_{0,-}$ also vanishes on $U^{\prime}$. In particular, $U^{\prime}$ satisfies Lemma 4.13 (iii) trivially. Moreover, since $\lambda$ is identically zero on $U^{\prime}$, the function $\mu$ of Lemma 4.13 necessarily takes values in $\mathcal{H}_{2}^{1}$. Since $\mu$ is non-zero on $U \subset U^{\prime}$, Lemma 4.14 implies that $U \cdot o=U^{\prime} \cdot o \subset X$ is a non-trivial integral of the Schubert system.

\subsection{Proof of Theorem 4.1 in the case that $H_{1}$ fails}

We will follow the strategy outlined in Section 4.1. Suppose that $\mathrm{H}_{1}$ fails for a pair $\gamma,-\beta$; see Definition 4.6. Then [19, Lemma 5.28] asserts that

$$
\mathfrak{g}_{-\beta} \otimes \mathfrak{g}_{\gamma} \subset \mathcal{H}_{1}^{1}
$$


Lemma 3.17 and the fact that $\gamma \in \Delta\left(\mathfrak{g}_{1, a}\right)$ is a $\mathfrak{g}_{0,0}$-highest weight yield $w^{\prime} \in W^{\mathfrak{p}}$ such that $\Delta\left(w^{\prime}\right) \sqcup\{\gamma\}=\Delta(w)$. Fix $0 \neq C \in \mathfrak{g}_{-\gamma}$ and $0 \neq B \in \mathfrak{g}_{-\beta}$. Let $\mathfrak{a}=\langle B+C\rangle$.

Claim. The direct sum $\mathfrak{n}^{\prime}:=\mathfrak{a} \oplus \mathfrak{n}_{w^{\prime}}$ is a subalgebra of $\mathfrak{g}$.

The claim is proved at the end of this section. Assuming the claim holds, let $N^{\prime}=\exp \left(\mathfrak{n}^{\prime}\right) \subset G$. When restricted to $N^{\prime}$ the Maurer-Cartan form $\vartheta$ takes values in $\mathfrak{n}^{\prime}$. In particular, $U=N^{\prime}$ satisfies hypotheses (i) and (ii) of Lemma 4.15. Moreover, the map $\lambda$ of Lemma 4.15 is the non-zero, constant map given by $\lambda_{\mid \mathfrak{n}_{w^{\prime}}}=0$ and $\lambda(C)=B$. By (4.16), $\lambda$ lies in $\mathcal{H}_{1}^{1}$. It follows from Lemma 4.15.a that $N^{\prime} \cdot o \subset X$ is a non-trivial integral manifold of the Schubert system.

Finally, note that $\mathfrak{n}^{\prime} \subset \mathfrak{g}_{-1} \oplus \mathfrak{g}_{0,-1}$ is nilpotent. It follows that $N^{\prime}=$ $\exp \left(\mathfrak{n}^{\prime}\right)$ is a linear algebraic subgroup of $G$. Therefore, the Zariski closure $Y_{1}=\overline{N^{\prime} \cdot o}$ is an irreducible algebraic subvariety of $X$ with $\operatorname{dim} Y_{1}=\operatorname{dim}$ $N^{\prime}=\operatorname{dim} X_{w}$. Therefore, $Y_{1}$ is a non-trivial, integral variety of the Schubert system. Modulo the claim, this completes the proof of Theorem 4.1 in the case that $\mathrm{H}_{1}$ fails.

Remark 4.17. Let $A=\exp (\mathfrak{a})$. Then $\overline{N^{\prime}}=\overline{A N_{w^{\prime}}}$ and $Y_{1}=\overline{A \cdot X_{w^{\prime}}}$.

The proof the the claim makes use of the following

Remark 4.18. Given two roots $\mu, \nu \in \Delta$, we have $\left[\mathfrak{g}_{\mu}, \mathfrak{g}_{\nu}\right]=\mathfrak{g}_{\mu+\nu}$. In particular, $\mu+\nu \in \Delta$ if and only if $[\zeta, \xi] \neq 0$ for every $0 \neq \zeta \in \mathfrak{g}_{\mu}$ and $0 \neq \xi \in$ $\mathfrak{g}_{\nu}$. See, for example, [15, Corollary 2.35].

Proof of claim. Since both $\mathfrak{a}$ and $\mathfrak{n}_{w^{\prime}}$ are subalgebras of $\mathfrak{g}$, it suffices to show that $\left[\mathfrak{a}, \mathfrak{n}_{w^{\prime}}\right] \subset \mathfrak{n}^{\prime}$. To that end, let $\xi \in \mathfrak{n}_{w^{\prime}}$. Then $C \in \mathfrak{g}_{-\gamma} \subset \mathfrak{g}_{-1}$ and Remark 3.3 yield

$$
[B+C, \xi]=[B, \xi]
$$

Without loss of generality we may assume that $\xi \in \mathfrak{g}_{-\mu}$ is a non-zero root vector, $\mu \in \Delta\left(w^{\prime}\right)$. By Remark 4.18, $[B, \xi] \in \mathfrak{g}_{-\beta-\mu}$ is non-zero if and only if $\beta+\mu$ is a root; assume this is the case. We wish to show that $\mathfrak{g}_{-\beta-\mu} \subset \mathfrak{n}_{w^{\prime}}$; equivalently, $\beta+\mu \in \Delta\left(w^{\prime}\right)$. 
By Proposition 3.13, $\Delta(w)=\Delta\left(\mathfrak{g}_{1, \leq \mathrm{a}}\right)$. Since $\Delta\left(w^{\prime}\right) \sqcup\{\gamma\}=\Delta(w)$ and $\gamma \in \Delta\left(\mathfrak{g}_{1, \mathrm{a}}\right)$, we have

$$
0 \leq \mu\left(Z_{w}\right) \leq \mathrm{a} \quad \text { and } \quad \Delta\left(\mathfrak{g}_{1,<\mathrm{a}}\right) \subset \Delta\left(w^{\prime}\right) \subset \Delta\left(\mathfrak{g}_{1, \leq \mathrm{a}}\right)
$$

We consider three cases: first, if $\mu\left(Z_{w}\right)<\mathrm{a}-1$, then $(\mu+\beta)\left(Z_{w}\right)<\mathrm{a}$ and $\beta+\mu \in \Delta\left(w^{\prime}\right)$. Second, if $\mu\left(Z_{w}\right)=\mathrm{a}-1$, then $(\mu+\beta)\left(Z_{w}\right)=$ a. By (4.7.i), $\mu+\beta \neq \gamma$, so $\mu+\beta \in \Delta\left(w^{\prime}\right)$. Third, if $\mu\left(Z_{w}\right)=$ a, then (4.7.ii) implies $\beta+\mu$ is not a root.

\subsection{Proof of Theorem 4.1 in the case that $\mathrm{H}_{2}$ fails}

We will follow the strategy outlined in Section 4.1. Suppose that $\mathrm{H}_{2}$ fails for a pair $\gamma \in \Delta\left(\mathfrak{g}_{1, \mathrm{a}}\right)$ and $\varepsilon \in \Delta\left(\mathfrak{g}_{1, \mathrm{a}-1}\right)$, see Definition 4.6. Then [19, Lemma 5.39] asserts that

$$
\mathfrak{g}_{\varepsilon} \otimes \mathfrak{g}_{\gamma} \subset \mathcal{H}_{2}^{1}
$$

By Lemma 3.17 there exists $w^{\prime} \in W^{\mathfrak{p}}$ such that $\Delta\left(w^{\prime}\right) \sqcup\{\gamma\}=\Delta(w)$. Fix $0 \neq C \in \mathfrak{g}_{-\gamma}$ and $0 \neq E \in \mathfrak{g}_{\varepsilon}$. Set

$$
\mathfrak{a}:=\langle C+E\rangle \subset \mathfrak{g} \text { and } A:=\exp (\mathfrak{a}) \subset G
$$

We will show that $Y_{2}=\overline{A N_{w^{\prime}} \cdot o}$ is a non-trivial integral variety of the Schubert system.

Because $\exp : \mathfrak{g} \rightarrow G$ is a diffeomorphism from a neighborhood of $0 \in \mathfrak{g}$ to a neighborhood of $\operatorname{Id} \in G$, it follows that there exist connected neighborhoods $A_{0} \subset A$ and $N_{0} \subset N_{w^{\prime}}$ of Id that are embedded submanifolds in $G$. Let $\mathbf{m}: G \times G \rightarrow G$ denote the multiplication map. Then $\mathbf{m}_{*}: \mathfrak{g} \times \mathfrak{g} \rightarrow \mathfrak{g}$ is given by $\mathbf{m}_{*}(u, v)=u+v$. In particular, the restriction $\mathbf{m}_{*}: \mathfrak{a} \times \mathfrak{n}_{w^{\prime}} \rightarrow$ $\mathfrak{a} \oplus \mathfrak{n}_{w^{\prime}}$ is a bijection. It follows, shrinking $A_{0}$ and $N_{0}$ if necessary, that there is a neighborhood $U \subset G$ of Id such that $\mathbf{m}: A_{0} \times N_{0} \rightarrow U$ is an isomorphism.

Compute the Maurer-Cartan form $\vartheta$ on $U \subset G$ as follows. Let $g_{0} g_{1} \in U$ with $g_{0} \in A_{0}$ and $g_{1} \in N_{0}$. The isomorphism $\phi: G \rightarrow G$ defined by $\phi(g)=$ $g_{0} g g_{1}$ preserves $A N_{w^{\prime}}$ and so induces an isomorphism $\phi_{*}: \mathfrak{a} \oplus \mathfrak{n}_{w^{\prime}} \rightarrow T_{g_{0} g_{1}} U$. So any element of $T_{g_{0} g_{1}} U$ may be expressed as $\phi_{*} \xi$ with $\xi \in \mathfrak{a} \oplus \mathfrak{n}_{w^{\prime}}$. Given $g \in G$, let $L_{g}, R_{g}: G \rightarrow G$ denote the left- and right-multiplication maps. 
Then $\phi=L_{g_{0}} R_{g_{1}}$. By definition

$$
\vartheta\left(\phi_{*} \xi\right)=\left(L_{g_{0} g_{1}}^{-1}\right)_{*}\left(\phi_{*} \xi\right)=\operatorname{Ad}_{g_{1}^{-1}} \xi
$$

Therefore, $\vartheta_{\mid U}$ takes values in $\operatorname{Ad}_{N_{w^{\prime}}}\left(\mathfrak{a} \oplus \mathfrak{n}_{w^{\prime}}\right)=\operatorname{Ad}_{N_{w^{\prime}}}(\mathfrak{a}) \oplus \operatorname{Ad}_{N_{w^{\prime}}}\left(\mathfrak{n}_{w^{\prime}}\right)$. Since $\mathfrak{n}_{w^{\prime}}$ is an algebra, we have

$$
\operatorname{Ad}_{N_{w^{\prime}}}\left(\mathfrak{n}_{w^{\prime}}\right)=\mathfrak{n}_{w^{\prime}}
$$

It remains to consider

$$
\operatorname{Ad}_{N_{w^{\prime}}}(\mathfrak{a})=\operatorname{Ad}_{\exp \left(\mathfrak{n}_{w^{\prime}}\right)}(\mathfrak{a})=\left(\exp \circ \operatorname{ad}_{\mathfrak{n}_{w^{\prime}}}\right)(\mathfrak{a})
$$

The algebra $\mathfrak{a}$ is spanned by $C+E$. By Remark 3.3, $\left[\mathfrak{n}_{w^{\prime}}, C+E\right]=\left[\mathfrak{n}_{w^{\prime}}, E\right]$. Equations (3.14), (4.8), and $\mathfrak{n}_{w^{\prime}} \oplus \mathfrak{g}_{-\gamma}=\mathfrak{n}_{w}$ imply that $\left[\mathfrak{n}_{w^{\prime}}, E\right] \subset \mathfrak{g}_{0}$. Moreover, the fact that $\varepsilon \in \Delta\left(\mathfrak{g}_{1, a-1}\right)$ is a highest $\mathfrak{g}_{0,0}$-weight yields

$$
\left[\mathfrak{n}_{w^{\prime}}, E\right] \subset \mathfrak{h}_{\varepsilon} \oplus \mathfrak{g}_{0}^{+}, \quad \text { where } \mathfrak{h}_{\varepsilon}:=\left[\mathfrak{g}_{\varepsilon}, \mathfrak{g}_{-\varepsilon}\right] \quad \text { and } \quad \mathfrak{g}_{0}^{+}:=\oplus_{\alpha \in \Delta^{+}\left(\mathfrak{g}_{0}\right)} \mathfrak{g}_{\alpha}
$$

By Proposition 3.13, $\Delta\left(w^{\prime}\right) \sqcup\{\gamma\}=\Delta(w)=\Delta\left(\mathfrak{g}_{1, \leq \mathrm{a}}\right)$. So, since $\gamma \in \Delta\left(\mathfrak{g}_{1, \mathrm{a}}\right)$ is a highest $\mathfrak{g}_{0,0}$-weight, we have $\left[\mathfrak{n}_{w^{\prime}}, \mathfrak{h}_{\varepsilon} \oplus \mathfrak{g}_{0}^{+}\right] \subset \mathfrak{n}_{w^{\prime}}$. Therefore,

$$
\left(\exp \circ \operatorname{ad}_{\mathfrak{n}_{w^{\prime}}}\right)(\mathfrak{a}) \subset \mathfrak{a} \oplus\left(\mathfrak{h}_{\varepsilon} \oplus \mathfrak{g}_{0}^{+}\right) \oplus \mathfrak{n}_{w^{\prime}}
$$

Now (4.21) yields $\vartheta_{\mathfrak{n}_{w}^{\perp}}=0$ on $U$, and $\vartheta_{\mathfrak{n}_{w}}: T_{z} U \rightarrow \mathfrak{n}_{w}$ is a linear isomorphism for all $z \in U$. Lemma 4.15 implies $U \cdot o \subset X$ is an integral manifold of the Schubert system. Equations (4.21) also imply that $\vartheta_{0,-}$ vanishes on $U$. Similarly, (4.21) implies that $\vartheta_{1,<\mathrm{a}}=\mu\left(\vartheta_{\mathfrak{n}_{w}}\right)$, where $\mu: U \rightarrow \mathfrak{g}_{1,<\mathrm{a}} \otimes \mathfrak{n}_{w}^{*}$ is the non-zero, constant map defined by $\mu_{\mid \mathfrak{n}_{w^{\prime}}}=0$ and $\mu(C)=E$. By (4.19), $\mu$ takes values in $\mathcal{H}_{2}^{1}$. By Lemma $4.15(\mathrm{~b}), U \cdot o \subset X$ is a non-trivial integral manifold of the Schubert system.

Finally, to see that the Zariski closure

$$
Y_{2}=\overline{U \cdot o}=\overline{A N_{w^{\prime}} \cdot o}
$$

is a variety of dimension $|w|$, it suffices to observe that $A$ and $N_{w^{\prime}}$ are algebraic. This is a consequence of the fact that both $\mathfrak{a}$ and $\mathfrak{n}_{w^{\prime}}$ are nilpotent. The nilpotency of $\mathfrak{n}_{w^{\prime}}$ is immediate from Remark 3.3. To see that $\mathfrak{a}$ is nilpotent, note that $\left[\mathfrak{a}, \mathfrak{g}_{ \pm 1}\right] \subset \mathfrak{g}_{0}$ and $\operatorname{ad}_{\mathfrak{a}}^{2}\left(\mathfrak{g}_{0, \ell}\right) \subset \mathfrak{g}_{0, \ell-1}$; these relations imply that the adjoint action of $\mathfrak{a}$ on $\mathfrak{g}$ is nilpotent. This completes the proof of Theorem 4.1. 


\section{Acknowledgments}

Over the course of this project, I have benefitted from conversations and/or correspondence with many people, including R. Bryant, I. Coskun, J.M. Landsberg, N. Ressayre, F. Sottile and D. The. I thank them for their insights and time. I am especially grateful to the anonymous referees for several ameliorating suggestions. This work was partially supported by NSF DMS-1006353.

\section{Appendix A. Geometric versus representation theoretic descriptions}

In this section, we provide the 'dictionary' between the representation theoretic (a, J)-description of Schubert varieties (Section 3.4) used in the proof of Theorem 4.1 and the more familiar and geometric, partition-based descriptions for the classical $G / P$. This dictionary, applied to Corollary 4.3 and [19, Theorem 6.1], yields the theorems of Section 2.

\section{A.1. Notation}

Given a vector space $V \simeq \mathbb{C}^{n}$, we fix a basis $\left\{e_{1}, \ldots, e_{n}\right\}$. Let $\left\{e^{1}, \ldots, e^{n}\right\}$ denote the dual basis of $V^{*}$. Set

$$
e_{\ell}^{k} \stackrel{\text { dfn }}{=} e_{\ell} \otimes e^{k} \in \operatorname{End}(V) \quad \text { for all } \leq k, \quad \ell \leq n
$$

Remark A.1. I follow the notation of [19], with the following exception. In [19], we uniformly write $J=\left\{j_{1}<\cdots<j_{p}\right\}$. In this paper, it is convenient to reorder the $j_{\ell}$ in some cases.

\section{A.2. Odd-dimensional quadrics $Q^{2 n-1}=B_{n} / P_{1}$}

Set $m=2 n-1$. There is a bijection between $W^{\mathfrak{p}} \backslash\left\{1, w_{0}\right\}$ and pairs a, J such that $\mathrm{J}=\{\mathrm{j}\} \subset\{2, \ldots, n\}$ and $\mathrm{a} \in\{0,1\}$; see [19, Corollary 3.17]. The Schubert variety $X_{w}$ associated with a fixed a, J may be described as follows.

Given a non-degenerate symmetric quadric form $(\cdot, \cdot)$ on $\mathbb{C}^{2 n+1}$, fix a basis $\left\{e_{1}, \ldots, e_{2 n+1}\right\}$ of $\mathbb{C}^{2 n+1}$ so that $\left(e_{k}, e_{\ell}\right)=\left(e_{n+k}, e_{n+\ell}\right)=\left(e_{k}, e_{2 n+1}\right)=$ $\left(e_{n+k}, e_{2 n+1}\right)=0,\left(e_{k}, e_{n+\ell}\right)=\delta_{k \ell}$, for all $1 \leq k, \ell \leq n$, and $\left(e_{2 n+1}, e_{2 n+1}\right)=$ 
1. The abelian subalgebra $\mathfrak{g}_{-1}$ is spanned by the root vectors

$$
\begin{array}{rll}
e_{k}^{1}-e_{n+1}^{n+k}, & \text { with root } & -\left(\alpha_{1}+\cdots+\alpha_{k-1}\right), \\
e_{n+k}^{1}-e_{n+1}^{k}, & \text { with root } & -\left(\alpha_{1}+\cdots+\alpha_{k-1}+2\left(\alpha_{k}+\cdots+\alpha_{n}\right)\right), \\
e_{2 n+1}^{1}-e_{n+1}^{2 n+1}, & \text { with root } & -\left(\alpha_{1}+\cdots+\alpha_{n}\right),
\end{array}
$$

$2 \leq k \leq n$.

Set $o=\left[e_{1}\right] \in \mathbb{P}^{2 n}$. If $\mathrm{a}=0$, then

$$
\mathfrak{n}_{w}=\left\langle e_{k}^{1}-e_{n+1}^{n+k} \mid k \leq j\right\rangle \quad \text { and } \quad X_{w}=\overline{\exp \left(\mathfrak{n}_{w}\right) \cdot o}=\mathbb{P}\left\langle e_{1}, \ldots, e_{j}\right\rangle=\mathbb{P}^{j-1} .
$$

If $\mathrm{a}=1$, then

$$
\mathfrak{n}_{w}=\left\langle e_{k}^{1}-e_{n+1}^{n+k}, e_{n+\ell}^{1}-e_{n+1}^{\ell}, e_{2 n+1}^{1}-e_{n+1}^{2 n+1} \mid 1 \leq k \leq n, j<\ell\right\rangle
$$

and $X_{w}=\overline{\exp \left(\mathfrak{n}_{w}\right) \cdot o}=Q^{m} \cap \mathbb{P}\left\langle e_{1}, \ldots, e_{n+1}, e_{n+j+1}, \ldots, e_{2 n+1}\right\rangle$.

\section{A.3. Even-dimensional quadrics $Q^{2 n-2}=D_{n} / P_{1}$}

Set $m=2 n-2$. There is a bijection between $W^{\mathfrak{p}} \backslash\left\{1, w_{0}\right\}$ and pairs a, J such that either

$\circ \mathrm{a}=0$ and $\mathrm{J}=\{\mathrm{j}\} \subset\{2, \ldots, n\}$ or $\mathrm{J}=\{n-1, n\}$; or

$\circ \mathrm{a}=1$ and $\mathrm{J}=\{\mathrm{j}\} \subset\{2, \ldots, n-2\}$ or $\mathrm{J}=\{n-1, n\}$.

See [19, Corollary 3.17]. The Schubert variety $X_{w}$ associated with a fixed a, J may be described as follows.

Given a non-degenerate symmetric quadric form $(\cdot, \cdot)$ on $\mathbb{C}^{2 n}$, fix a basis $\left\{e_{1}, \ldots, e_{2 n}\right\}$ of $\mathbb{C}^{2 n}$ so that $\left(e_{k}, e_{\ell}\right)=\left(e_{n+k}, e_{n+\ell}\right)=0$ and $\left(e_{k}, e_{n+\ell}\right)=\delta_{k \ell}$, for all $1 \leq k, \ell \leq n$. The abelian subalgebra $\mathfrak{g}_{-1}$ is spanned by the root vectors

$$
\begin{array}{rrl}
e_{k}^{1}-e_{n+1}^{n+k}, \quad \text { with root } & -\left(\alpha_{1}+\cdots+\alpha_{k-1}\right), \\
e_{n+\ell}^{1}-e_{n+1}^{\ell}, \quad \text { with root } & -\left(\alpha_{1}+\cdots+\alpha_{\ell-1}+2\left(\alpha_{\ell}+\cdots+\alpha_{n-2}\right)\right. \\
& \left.+\alpha_{n-1}+\alpha_{n}\right), \\
e_{2 n}^{1}-e_{n+1}^{n}, \quad \text { with root } & -\left(\alpha_{1}+\cdots+\alpha_{n-2}\right)-\alpha_{n},
\end{array}
$$

with $2 \leq k \leq n$ and $2 \leq \ell \leq n-1$. 
Set $o=\left[e_{1}\right] \in \mathbb{P} \mathbb{C}^{2 n}$. First suppose that $\mathrm{a}=0$. If $\mathrm{J}=\{\mathrm{j}\}$ with $2 \leq \mathrm{j} \leq$ $n-2$, then $X_{w}=\mathbb{P}^{\mathrm{j}-1}$. If $\mathrm{J}=\{n-1\}$ or $\mathrm{J}=\{n\}$, then $X_{w}=\mathbb{P}^{n-1}$. If $\mathrm{J}=$ $\{n-1, n\}$, then $X_{w}=\mathbb{P}^{n-2}$.

Next suppose that $\mathrm{a}=1$. If $\mathrm{J}=\{\mathrm{j}\}$ with $2 \leq \mathrm{j} \leq n-2$, then $X_{w}=$ $Q^{m} \cap \mathbb{P}\left\langle e_{1}, \ldots, e_{n+1}, e_{n+j+1}, \ldots, e_{2 n}\right\rangle$. If $\mathrm{J}=\{n-1, n\}$, then $X_{w}=Q^{m} \cap$ $\mathbb{P}\left\langle e_{1}, \ldots, e_{n+1}, e_{2 n}\right\rangle$.

\section{A.4. Grassmannians $\operatorname{Gr}(\mathrm{i}, n+1)=A_{n} / P_{\mathrm{i}}$}

There is a bijection between $W^{\mathfrak{p}} \backslash\left\{1, w_{0}\right\}$ and pairs a, $\mathrm{J}$ such that $\mathrm{J}=\left\{\mathrm{j}_{\mathrm{p}}, \ldots\right.$, $\left.\mathrm{j}_{1}, \mathrm{k}_{1}, \ldots, \mathrm{k}_{\mathrm{q}}\right\} \subset\{1, \ldots, n\} \backslash\{\mathrm{i}\}$ is ordered so that

$$
1 \leq \mathrm{j}_{\mathrm{p}}<\cdots<\mathrm{j}_{1}<\mathrm{i}<\mathrm{k}_{1}<\cdots<\mathrm{k}_{\mathrm{q}} \leq n
$$

and satisfying $\mathrm{p}, \mathrm{q} \in\{\mathrm{a}, \mathrm{a}+1\}$; see [19, Corollary 3.17]. (Beware, these $\mathrm{p}, \mathrm{q}$ do not agree with those of [19], cf. Remark A.1.) For convenience we set

$$
\mathrm{j}_{\mathrm{p}+1}:=0, \quad \mathrm{j}_{0}:=\mathrm{i}=: \mathrm{k}_{0}, \quad \mathrm{k}_{\mathrm{q}+1}:=n+1 .
$$

Given a, J, the corresponding Schubert variety $X_{w} \subset \operatorname{Gr}(\mathbf{i}, n+1)$ is described as follows. Fix a basis $\left\{e_{1}, \ldots, e_{n+1}\right\}$ of $\mathbb{C}^{n+1}$. The abelian subalgebra $\mathfrak{g}_{-1}$ is spanned by the root vectors $\left\{e_{\ell}^{k} \mid 1 \leq k \leq i<\ell \leq n+1\right\}$; the corresponding roots are $-\left(\alpha_{k}+\cdots+\alpha_{\ell-1}\right)$. Define a filtration $\mathrm{F}_{\mathrm{a}+1} \subset \mathrm{F}_{\mathrm{a}} \subset$ $\cdots \subset \mathrm{F}_{1} \subset \mathrm{F}_{0}$ of $\mathbb{C}^{n+1}$ by

$$
\mathrm{F}_{\ell}=\left\langle e_{1}, e_{2}, \ldots, e_{\mathbf{j} \ell}, e_{\mathbf{i}+1}, e_{\mathbf{i}+2}, \ldots, e_{\mathbf{k}_{m}}\right\rangle \quad \text { with } \ell+m=\mathrm{a}+1
$$

Set $o=\left[e_{1} \wedge \cdots \wedge e_{\mathbf{i}}\right] \in \mathbb{P}\left(\wedge^{\mathbf{i}} \mathbb{C}^{n+1}\right)$. Then

$$
X_{w}=\left\{E \in \operatorname{Gr}(\mathbf{i}, n+1) \mid \operatorname{dim}\left(E \cap \mathrm{F}_{\ell}\right) \geq \mathrm{j}_{\ell}, 0 \leq \ell \leq \mathrm{a}+1\right\} .
$$

Example A.3. Consider $X=\operatorname{Gr}(5,13) \simeq A_{12} / P_{5}$. The marking $\mathrm{J}=$ $\{2,3,7,9,12\}$ and integer $\mathrm{a}=2$ define the filtration $\mathrm{F}_{3} \subset \mathrm{F}_{2} \subset \mathrm{F}_{1} \subset \mathrm{F}_{0}$ as

$$
\begin{aligned}
& \mathrm{F}_{3}=\langle 0\rangle, \quad \mathrm{F}_{2}=\left\langle e_{1}, e_{2}, e_{6}, e_{7}\right\rangle, \\
& \mathrm{F}_{1}=\left\langle e_{1}, e_{2}, e_{3}, e_{6}, e_{7}, e_{8}, e_{9}\right\rangle, \quad \mathrm{F}_{0}=\left\langle e_{1}, \ldots, e_{5}, e_{6}, \ldots e_{12}\right\rangle .
\end{aligned}
$$

The associated Schubert variety is the set of all $E \in \operatorname{Gr}(5,13)$ such that

$$
\begin{aligned}
& \operatorname{dim}\left(E \cap \mathrm{F}_{3}\right) \geq 0, \quad \operatorname{dim}\left(E \cap \mathrm{F}_{2}\right) \geq 2, \\
& \operatorname{dim}\left(E \cap \mathrm{F}_{1}\right) \geq 3, \quad \operatorname{dim}\left(E \cap \mathrm{F}_{0}\right) \geq 5 .
\end{aligned}
$$




\section{Partitions versus a, J}

It is well-known that Schubert varieties in $X=\operatorname{Gr}(i, n+1)$ are indexed by partitions

(A.4) $\lambda=\left(\lambda_{1}, \ldots, \lambda_{i}\right) \in \mathbb{Z}^{i} \quad$ such that $1 \leq \lambda_{1}<\lambda_{2}<\cdots<\lambda_{i} \leq n+1$,

cf. [1, Section 3.1.3]. Fix a flag $0 \subset F^{1} \subset F^{2} \subset \cdots \subset F^{n+1}$. The corresponding Schubert variety is

$$
Y_{\lambda}\left(F^{\bullet}\right):=\left\{E \in X \mid \operatorname{dim}\left(E \cap F^{\lambda_{k}}\right) \geq k, \forall k\right\}
$$

Note that, if $\lambda_{k+1}=\lambda_{k}+1$, then the condition $\operatorname{dim}\left(E \cap F^{\lambda_{k}}\right) \geq k$ is redundant; it is implied by $\operatorname{dim}\left(E \cap F^{\lambda_{k+1}}\right) \geq k+1$. To remove the redundancies, decompose $\lambda=\mu_{\mathrm{p}} \cdots \mu_{1} \mu_{0}$ into maximal blocks of consecutive integers. For example, if $\lambda=(2,3,4,7,8,12)$, then $\mu_{2}=(2,3,4), \mu_{1}=(7,8)$ and $\mu_{0}=(12)$. Let

$$
j_{\ell}(\lambda)=\left|\mu_{\mathrm{p}} \cdots \mu_{\ell}\right|
$$

be the length of the subpartition $\mu_{\mathrm{p}} \cdots \mu_{\ell}$. (In all cases, $j_{0}=|\lambda|=i$.) In the preceding example, $\mathrm{j}_{2}=3, \mathrm{j}_{1}=5$ and $\mathrm{j}_{0}=6$.

Remark A.7. Define $\tilde{\lambda}_{k}=\lambda_{k}-k$. Then $0 \leq \tilde{\lambda}_{1} \leq \tilde{\lambda}_{2} \leq \cdots \leq \tilde{\lambda}_{n} \leq n$. Condense $\tilde{\lambda}$ by writing $\tilde{\lambda}=\left(\nu_{1}^{c_{1}}, \ldots, \nu_{t}^{c_{t}}\right)$ with $0 \leq \nu_{1}<\nu_{2}<\cdots \nu_{t} \leq n$ and $0<c_{i}$. Then, $t=\mathrm{p}+1$, and $\mathrm{j}_{\mathrm{p}}=c_{1}, \mathrm{j}_{\mathrm{p}-1}=c_{1}+c_{2}, \mathrm{j}_{\mathrm{p}-2}=c_{1}+c_{2}+c_{3}$, et cetera: $\mathrm{j}_{\mathrm{p}+1-s}=c_{1}+\cdots+c_{s}$, and $\mathrm{j}_{\mathrm{p}-s}-\mathrm{j}_{\mathrm{p}+1-s}=c_{s+1}$.

Note that $\lambda_{j_{\ell}}$ is the last entry in the block $\mu_{\ell}$. That is,

$$
\left\{\lambda_{\mathrm{j}_{\mathrm{p}}}, \ldots, \lambda_{\mathrm{j}_{1}}\right\}=\left\{\lambda_{k} \in \lambda \mid \lambda_{k}-\lambda_{k+1}>1,1 \leq k<\mathrm{i}\right\}
$$

The redundancy-free formulation of $(\mathrm{A} .5)$ is

$$
Y_{\lambda}\left(F^{\bullet}\right)=\left\{E \in X \mid \operatorname{dim}\left(E \cap F^{\lambda_{j_{\ell}}}\right) \geq \mathrm{j}_{\ell}, 1 \leq \ell \leq \mathrm{p}\right\}
$$

The following is [19, Proposition 3.30].

Lemma A.9 [19]. Let $\lambda=\left(\lambda_{1}, \ldots, \lambda_{\mathrm{i}}\right)$ be a partition satisfying (A.4), and let $\lambda=\mu_{\mathrm{p}} \ldots \mu_{1} \mu_{0}$ be the decomposition of $\lambda$ into maximal blocks of consecutive integers. The pair $\mathrm{a}, \mathrm{J}=\left\{\mathrm{j}_{\mathrm{p}}, \ldots, \mathrm{j}_{1}, \mathrm{k}_{1}, \ldots, \mathrm{k}_{\mathrm{q}}\right\}$ associated to the 
Schubert class $\xi_{\lambda}$ is given by (A.6),

$$
\left\{\mathrm{k}_{1}, \ldots, \mathrm{k}_{\mathrm{q}}\right\}=\left\{\mathrm{i}-\mathrm{j}_{\mathrm{p}}+\lambda_{\mathrm{j}_{\mathrm{p}}}, \ldots, \mathrm{i}-\mathrm{j}_{1}+\lambda_{\mathrm{j}_{1}}, \lambda_{\mathrm{i}}\right\} \backslash\{\mathrm{i}, n+1\}
$$

and

$$
\mathrm{a}=\left\{\begin{array}{ll}
\mathrm{p} & \text { if } \lambda_{1}>1 \\
\mathrm{p}-1 & \text { if } \lambda_{1}=1
\end{array}\right\}= \begin{cases}\mathrm{q}, & \text { if } \lambda_{\mathrm{i}}=n+1 \\
\mathrm{q}-1, & \text { if } \lambda_{\mathrm{i}}<n+1 .\end{cases}
$$

Conversely, given $\mathrm{a}, \mathrm{J}$, the associated partition $\lambda=\mu_{\mathrm{p}} \ldots \mu_{1} \mu_{0}$ is given by

$$
\mu_{\ell}=\left(\mathrm{j}_{\ell+1}+\mathrm{k}_{m}-\mathrm{i}+1, \ldots, \mathrm{j}_{\ell}+\mathrm{k}_{m}-\mathrm{i}\right)
$$

with $\ell+m=\mathrm{a}+1$.

Example A.10. In Example A.3, we have $\lambda=(3,4,7,11,12)$.

Remark A.11. Together [19, Proposition 3.30] and [1, Theorem 9.3.1] imply that the integer $\mathrm{a}(w)$ is the number of irreducible components of $\operatorname{Sing}\left(X_{w}\right)$. The relationship between $\mathrm{a}(w)$ and the number of irreducible components in $\operatorname{Sing}\left(X_{w}\right)$ for the other (Lagrangian Grassmannian, Spinor variety, Cayley plane and Freudenthal variety) irreducible, cominuscule $X$ is given by [18, Corollary 3.6 and Table 1].

\section{A.5. Lagrangian Grassmannians $\mathrm{LG}(n, 2 n)=C_{n} / P_{n}$}

There exists a bijection between $W^{\mathfrak{p}} \backslash\left\{1, w_{0}\right\}$ and pairs $\mathrm{a} \geq 0$ and $\mathrm{J}=$ $\left\{j_{p}, \ldots, j_{1}\right\} \subset\{1, \ldots, n-1\}$ satisfying

$$
1 \leq j_{p}<\cdots<j_{1} \leq n-1
$$

and

$$
p \in\{a, a+1\}
$$

see [19, Corollary 3.17]. (These $j \ell$ have the opposite order of those in [19].) For convenience we set

$$
j_{\mathrm{p}+1}:=0, \quad j_{0}:=n .
$$

To describe the Schubert variety $X_{w}$, fix a non-degenerate skewsymmetric bilinear form $(\cdot, \cdot)$ on $\mathbb{C}^{2 n}$, and basis $\left\{e_{1}, \ldots, e_{2 n}\right\}$ of $\mathbb{C}^{2 n}$ satisfying $\left(e_{a}, e_{b}\right)=0=\left(e_{n+a}, e_{n+b}\right)$ and $\left(e_{a}, e_{n+b}\right)=\delta_{a b}$ for all $1 \leq a, b \leq n$. Then $X=\operatorname{LG}(n, 2 n)$ is the $C_{n}$-orbit of $\left\langle e_{1}, \ldots, e_{n}\right\rangle$. The abelian subalgebra 
$\mathfrak{g}_{-1}$ (which may be identified with $n$-by- $n$ symmetric matrices) is spanned by the root vectors $\left\{e_{n+k}^{j}+e_{n+j}^{k} \mid 1 \leq j \leq k \leq n\right\}$, with roots $-\left(\alpha_{j}+\cdots+\right.$ $\left.\alpha_{k-1}\right)-2\left(\alpha_{k}+\cdots+\alpha_{n}\right)$. Define a filtration $\mathrm{F}_{\mathrm{p}} \subset \cdots \subset \mathrm{F}_{1} \subset \mathrm{F}_{0}$ of $\mathbb{C}^{2 n}$ by

$$
\mathrm{F}_{\ell}=\left\langle e_{1}, \ldots, e_{j_{\ell}}, e_{n+j_{m}+1}, \ldots, e_{2 n}\right\rangle
$$

with

$$
\ell+m=\mathrm{a}+1 .
$$

Set $o=\left[e_{1} \wedge \cdots \wedge e_{n}\right] \in \mathbb{P}\left(\wedge^{n} \mathbb{C}^{2 n}\right)$. Then

$$
X_{w}=\left\{E \in X \mid \operatorname{dim}\left(E \cap \mathrm{F}_{\ell}\right) \geq \mathrm{j} \ell, \forall 0 \leq \ell \leq \mathrm{p}\right\} .
$$

Example A.17. Consider $X=\mathrm{LG}(5,10) \simeq C_{5} / P_{5}$. The marking $\mathrm{J}=$ $\{1,2,4\}$ and integer $\mathrm{a}=3$ define the filtration $\mathrm{F}_{3} \subset \mathrm{F}_{2} \subset \mathrm{F}_{1} \subset \mathrm{F}_{0}$ of $\mathbb{C}^{10}$ as

$$
\begin{aligned}
& \mathrm{F}_{3}=\left\langle e_{1}, e_{10}\right\rangle, \quad \mathrm{F}_{2}=\left\langle e_{1}, e_{2}, e_{8}, e_{9}, e_{10}\right\rangle, \\
& \mathrm{F}_{1}=\left\langle e_{1}, \ldots, e_{4}, e_{7}, \ldots, e_{10}\right\rangle, \quad \mathrm{F}_{0}=\mathbb{C}^{10} .
\end{aligned}
$$

The associated Schubert variety is the set of all $E \in \mathrm{LG}(5,10)$ such that

$$
\operatorname{dim}\left(E \cap F_{3}\right) \geq 1, \quad \operatorname{dim}\left(E \cap F_{2}\right) \geq 2, \quad \operatorname{dim}\left(E \cap F_{1}\right) \geq 4 .
$$

\section{Partitions versus a, $\mathrm{J}$}

It is well-known that Schubert varieties in $X=\mathrm{LG}(n, 2 n)$ are indexed by partitions $\lambda=\left(\lambda_{1}, \lambda_{2}, \ldots, \lambda_{n}\right)$ such that

$$
\begin{gathered}
1 \leq \lambda_{1}<\lambda_{2}<\cdots<\lambda_{n} \leq 2 n, \quad \text { and } \\
\lambda_{i} \in \lambda \quad \text { if and only if } 2 n+1-\lambda_{i} \notin \lambda,
\end{gathered}
$$

cf. [1, Section 9.3]. Fix an isotropic flag $F^{\bullet}$ in $\mathbb{C}^{2 n}$ by specifying $F^{k}=$ $\left\langle e_{1}, \ldots, e_{k}\right\rangle$ and $\left(F^{k}, F^{2 n-k}\right)=0$ for $1 \leq k \leq n$. The corresponding Schubert variety is given by (A.5). As was the case in Section A.4, this formulation is redundant. Again, to remove the redundancies, decompose $\lambda=\mu_{\mathrm{p}} \ldots \mu_{1} \mu_{0}$ into maximal blocks of consecutive integers. Let $j_{\ell}(\lambda)=\left|\mu_{\mathrm{p}} \ldots \mu_{\ell}\right|$ be the length of the subpartition $\mu_{\mathrm{p}} \ldots \mu_{s}$. (In all cases, $j_{0}=n$.) Then the redundancy-free formulation is (A.8). A comparison of this with (A.16) yields $\mathrm{J}=\left\{\mathrm{j}_{\mathrm{p}}(\lambda), \ldots, \mathrm{j}_{1}(\lambda)\right\}$, and $\lambda_{\mathrm{j}_{\ell}}=\operatorname{dim}_{\ell}=\mathrm{j}_{\ell}+n-\mathrm{j}_{m}$, for $\ell+m=$ $\mathrm{a}+1$; note that $\lambda_{\mathrm{j}_{\ell}}+\lambda_{\mathrm{j}_{m}}=2 n$. In order to determine the value of $\mathrm{a}$, 
we consider two cases:

$\circ$ First, suppose that $\lambda_{1}>1$. By (A.18b), this is equivalent to $\lambda_{n}=\lambda_{j_{0}}=$ 2n. Additionally, (A.18b) yields $\lambda_{j_{\ell}}+\lambda_{j_{m}}=2 n$ when $\ell+m=\mathrm{p}+1$. Therefore, $\mathrm{a}=\mathrm{p}$.

$\circ$ Second, suppose that $\lambda_{1}=1$, equivalently, $\lambda_{n}=\lambda_{j_{0}}<2 n$. Equation (A.18b) implies $\lambda_{j_{\ell}}+\lambda_{j_{m}}=2 n$ when $\ell+m=\mathrm{p}$. Thus, $\mathrm{p}=\mathrm{a}+1$.

As an example, Table 1 lists the partitions $\lambda$ and corresponding a : J values for the Schubert varieties in $\operatorname{LG}(5,10)$.

The preceding discussion may be summarized as follows.

Lemma A.19. Let $\lambda=\left(\lambda_{1}, \ldots, \lambda_{n}\right)$ be a partition satisfying (A.18). Let $\lambda=\mu_{\mathrm{p}} \ldots \mu_{1} \mu_{0}$ be a decomposition of $\lambda$ into $\mathrm{p}+1$ maximal blocks of consecutive integers. Then $\mathrm{J}(\lambda)=\left\{\mathrm{j}_{\mathrm{p}}(\lambda), \ldots, \mathrm{j}_{1}(\lambda)\right\}$ is given by (A.6), and

$$
a(\lambda)= \begin{cases}p-1 & \text { if } \lambda_{1}=1 \\ p & \text { if } \lambda_{1}>1\end{cases}
$$

Conversely, given a and $\mathrm{J}=\left\{\mathrm{j}_{\mathrm{p}}, \ldots, \mathrm{j}_{1}\right\}$ we construct $\lambda(\mathrm{a}, \mathrm{J})=\mu_{\mathrm{p}}(\mathrm{a}, \mathrm{J}) \ldots$ $\mu_{0}(\mathrm{a}, \mathrm{J})$ by

$$
\mu_{\ell}(\mathrm{a}, \mathrm{J})=\left(n+1+\mathrm{j}_{\ell+1}-\mathrm{j}_{m}, \ldots, n+\mathrm{j}_{\ell}-\mathrm{j}_{m}\right),
$$

with $\ell+m=\mathrm{a}+1$.

\section{A.6. Spinor varieties $\mathcal{S}_{n}=D_{n} / P_{n}$}

Given $\mathrm{a}=\mathrm{a}(w)$ and $\mathrm{J}=\mathrm{J}(w)$, note that

$$
\alpha_{n-1}\left(Z_{w}\right)=0 \text { if } n-1 \notin \mathrm{J}, \quad \text { and } \quad \alpha_{n-1}\left(Z_{w}\right)=1 \text { if } n-1 \in \mathrm{J} \text {. }
$$

Define

$$
r=\left\lceil\frac{1}{2}\left(\mathrm{a}+\alpha_{n-1}\left(Z_{w}\right)\right)\right\rceil= \begin{cases}\lceil\mathrm{a} / 2\rceil & \text { if } n-1 \notin \mathrm{J} \\ \lfloor\mathrm{a} / 2\rfloor+1 & \text { if } n-1 \in \mathrm{J}\end{cases}
$$

see also (A.28). There exists a bijection between $W^{\mathfrak{p}} \backslash\left\{1, w_{0}\right\}$, and pairs a $\geq 0$ and $\mathrm{J}=\left\{\mathrm{j}_{\mathrm{p}}, \ldots, \mathrm{j}_{1}\right\} \subset\{1, \ldots, n-1\}$, ordered by (A.12) and satisfying

$$
\mathrm{p}-\alpha_{n-1}\left(Z_{w}\right) \in\{\mathrm{a}, \mathrm{a}+1\}, \text { and } 2 \leq \mathrm{j}_{\mathrm{r}}-\mathrm{j}_{\mathrm{r}+1} \text { when } \mathrm{r}>\alpha_{n-1}\left(Z_{w}\right) \text {; }
$$


see [19, Corollary 3.17]. (These $j_{\ell}$ have the opposite order of those in [19].) We maintain the convention (A.13).

To describe the Schubert variety $X_{w}$, fix a non-degenerate symmetric bilinear form $(\cdot, \cdot)$ on $\mathbb{C}^{2 n}$ and basis $\left\{e_{1}, \ldots, e_{2 n}\right\}$ of $\mathbb{C}^{2 n}$ satisfying $\left(e_{a}, e_{b}\right)=$ $0=\left(e_{n+a}, e_{n+b}\right)$ and $\left(e_{a}, e_{n+b}\right)=\delta_{a b}$ for all $1 \leq a, b \leq n$. Our convention is that $\mathcal{S}_{n}$ is the $D_{n}$-orbit of $\left\langle e_{1}, \ldots, e_{n}\right\rangle$. The abelian subalgebra $\mathfrak{g}_{-1}$ (which may be identified with $n$-by- $n$ skew-symmetric matrices) is spanned by root vectors $\left\{e_{n+k}^{j}-e_{n+j}^{k} \mid 1 \leq j<k \leq n\right\}$. The corresponding roots are $-\left(\alpha_{j}+\right.$ $\left.\cdots+\alpha_{n-2}\right)-\alpha_{n}$, if $k=n$; $-\left(\alpha_{j}+\cdots+\alpha_{n}\right)$, if $k=n-1$; and $-\left(\alpha_{j}+\cdots+\right.$ $\left.\alpha_{k-1}\right)-2\left(\alpha_{k}+\cdots+\alpha_{n-2}\right)-\alpha_{n-1}-\alpha_{n}$, if $k<n-1$. Define a filtration $\mathrm{F}_{\mathrm{p}} \subset \cdots \subset \mathrm{F}_{1} \subset \mathrm{F}_{0}$ of $\mathbb{C}^{2 n}$ by (A.14) with

$$
\ell+m=\left\{\begin{array}{cc}
\mathrm{a}+1 & \text { if } n-1 \notin \mathrm{J}, \\
\mathrm{a}+2 & \text { if } n-1 \in \mathrm{J}
\end{array}\right\}=\mathrm{a}+1+\alpha_{n-1}\left(Z_{w}\right)
$$

Set $o=\left[e_{1} \wedge \cdots \wedge e_{n}\right] \in \mathbb{P}\left(\wedge^{n} \mathbb{C}^{2 n}\right)$. Then the Schubert variety $X_{w}$ is given by (A.16).

\section{Partitions versus a, J}

It is well-known that the Schubert varieties of $X=\mathcal{S}_{n}$ are indexed by partitions $\lambda=\left(\lambda_{1}, \lambda_{2}, \ldots, \lambda_{n}\right)$ satisfying (A.18) and

$$
\#\left\{i \mid \lambda_{i}>n\right\} \quad \text { is even, }
$$

cf. [1, Section 9.3]. Fix an isotropic flag $F^{\bullet}$ in $\mathbb{C}^{2 n}$ by specifying $F^{k}=$ $\left\langle e_{1}, \ldots, e_{k}\right\rangle$ and $\left(F^{k}, F^{2 n-k}\right)=0$ for $1 \leq k \leq n$. The corresponding Schubert variety is given by $(\mathrm{A} .5)$.

We define $\mathrm{J}(\lambda)$ as in (A.6), with the following modification of the block decomposition. In the block decomposition $\lambda=\hat{\mu}_{p} \ldots \hat{\mu}_{1} \hat{\mu}_{0}$, the integers $n-$ $1, n+1$ are considered 'consecutive' and are placed in the same $\hat{\mu}_{s}$-block; likewise, the integers $n, n+2$ are 'consecutive.' For example, if $n=5$, then $\lambda=(2,3,4,6,10)$ has block decomposition $\hat{\mu}_{1} \hat{\mu}_{0}=(2,3,4,6)(10)$; likewise, $\lambda=(1,2,5,7,8)$ has block decomposition $\hat{\mu}_{1} \hat{\mu}_{0}=(1,2)(5,7,8)$.

As before,

$$
j_{\ell}(\lambda)=\left|\hat{\mu}_{\mathrm{p}} \ldots \hat{\mu}_{\ell}\right|
$$


Define

(A.26)

$$
\mathrm{a}=\left\{\begin{array}{lll}
\mathrm{p}-2 & \text { if } \lambda_{1}=1 \quad \text { and } \quad \lambda_{n}-\lambda_{n-1}>1, \\
\mathrm{p}-1 & \text { if } \lambda_{1}=1 & \text { and } \quad \lambda_{n}-\lambda_{n-1}=1, \\
\mathrm{p} & \text { if } \lambda_{1}>1 & \text { and } \quad \lambda_{n}-\lambda_{n-1}=1 .
\end{array} \text { or } \lambda_{1}>1 \quad \text { and } \quad \lambda_{n}-\lambda_{n-1}>1\right.
$$

As an example, Table 2 lists the partitions and corresponding a : $\mathrm{J}$ and $r$ values for the Schubert varieties of $\mathcal{S}_{6}=\operatorname{Spin}_{12} \mathbb{C} / P_{6}$. The discussion above yields the following.

Lemma A.27. Given a partition $\lambda$ indexing a Schubert variety (A.5) in $\mathcal{S}_{n}=D_{n} / P_{n}$, the set $\mathrm{J}(\lambda)=\left\{\mathrm{j}_{\mathrm{p}}(\lambda), \ldots, \mathrm{j}_{1}(\lambda)\right\}$ is given by $(\mathrm{A} .25)$, and $\mathrm{a}(\lambda)$ is given by (A.26).

Conversely, given a and $\mathrm{J}=\left\{\mathrm{j}_{\mathrm{p}}, \ldots, \mathrm{j}_{1}\right\}$, we construct $\lambda(\mathrm{a}, \mathrm{J})$ as follows. Let $\lambda^{\prime}=\mu_{\mathrm{p}} \ldots \mu_{1} \mu_{0}$ be given by (A.20), with $\ell+m=\mathrm{a}+1+\alpha_{n-1}\left(Z_{w}\right)$. If $\lambda^{\prime}$ satisfies (A.24), then $\lambda=\lambda^{\prime}$. If (A.24) fails for $\lambda^{\prime}$, then we modify the partition as follows: precisely one of $\{n, n+1\}$ belongs to $\lambda^{\prime}$, denote this element by $a^{\prime}$, and the other by $a$. Then $\lambda$ is obtained from $\lambda^{\prime}$ by replacing $a^{\prime}$ with $a$.

Since $\mathrm{j}_{1}=n-1$ (equivalently, $\alpha_{n-1}\left(Z_{w}\right)=1$ ) if and only if $\lambda_{n}>\lambda_{n-1}+$ $1,($ A.26) is equivalent to

$$
\mathrm{a}(\lambda)+\alpha_{n-1}\left(Z_{w}\right)= \begin{cases}\mathrm{p}-1 & \text { if } \lambda_{1}=1 \\ \mathrm{p} & \text { if } \lambda_{1}>1\end{cases}
$$

Similarly, (A.21) may be expressed as

$$
r= \begin{cases}\lfloor p / 2\rfloor & \text { if } \lambda_{1}=1 \\ \lceil p / 2\rceil & \text { if } \lambda_{1}>1\end{cases}
$$

\section{References}

[1] S. Billey and V. Lakshmibai, Singular loci of Schubert varieties, Progress in Mathematics 182. Birkhäuser Boston Inc., Boston, MA, 2000.

[2] A. Borel and A. Haefliger, La classe d'homologie fondamentale d'un espace analytique, Bull. Soc. Math. France, 89 (1961), 461-513.

[3] R.L. Bryant, Rigidity and quasi-rigidity of extremal cycles in Hermitian symmetric spaces, Annals of Mathematics Studies, 153, Princeton University Press, 2010. arXiv:math/0006186. 
[4] R.L. Bryant, Metrics with exceptional holonomy, Ann. Math. (2) 126(3) (1987), 525-576.

[5] A. Čap and J. Slovák, Parabolic geometries. I, Mathematical Surveys and Monographs 154. American Mathematical Society, Providence, RI, 2009. Background and general theory.

[6] I. Coskun, Rigidity of Schubert classes in orthogonal Grassmannians, Israel J. Math. to appear.

[7] I. Coskun, Rigid and non-smoothable Schubert classes, J. Differ. Geom., 87(3) (2011), 493-514.

[8] I. Coskun and C. Robles, Flexibility of Schubert classes, Differ. Geom. Appl. (2013), arXiv:1303.0253.

[9] R. Hartshorne, E. Rees and E. Thomas, Nonsmoothing of algebraic cycles on Grassmann varieties, Bull. Amer. Math. Soc. 80 (1974), 847-851.

[10] H. Hironaka, Smoothing of algebraic cycles of small dimensions, Amer. J. Math. 90 (1968), 1-54.

[11] J. Hong, Rigidity of singular Schubert varieties in $\operatorname{Gr}(m, n)$, J. Differ. Geom. 71(1) (2005), 1-22.

[12] J. Hong, Rigidity of smooth Schubert varieties in Hermitian symmetric spaces, Trans. Amer. Math. Soc. 359(5) (2007), 2361-2381 (electronic).

[13] S.L. Kleiman, Geometry on Grassmannians and applications to splitting bundles and smoothing cycles, Inst. Hautes Études Sci. Publ. Math. (36) (1969), 281-297.

[14] S.L. Kleiman and J. Landolfi, Singularities of special Schubert varieties, in Symposia Mathematica, Vol. V (INDAM, Rome, 1969/70), Academic Press, London, 1969/1970, 341-346.

[15] A.W. Knapp, Lie groups beyond an introduction, volume 140 of Progress in Mathematics. Birkhäuser Boston Inc., Boston, MA, 2nd edn., 2002 .

[16] B. Kostant, Lie algebra cohomology and the generalized Borel-Weil theorem, Ann. Math. (2), 74 (1961), 329-387.

[17] B. Kostant, Lie algebra cohomology and generalized Schubert cells, Ann. Math. (2) 77 (1963), 72-144. 
[18] C. Robles, Singular loci of cominuscule Schubert varieties, J. Pure Appl. Algebra (2013), http://dx/doi.org/10.1016/j.jpaa.2013.08.014

[19] C. Robles and D. The, Rigid Schubert varieties in compact Hermitian symmetric spaces, Selecta Math. (N.S.), 18(3) (2012), 717-777.

[20] H. Thomas and A. Yong, A combinatorial rule for (co)minuscule Schubert calculus, Adv. Math. 222(2) (2009), 596-620.

[21] M. Walters, Geometry and uniqueness of some extreme subvarieties in complex Grassmannians, PhD thesis, University of Michigan, 1997.

Mathematics Department

MAIL-STOP 3368

TeXas A\&M University

College Station

TX 77843-3368

USA

E-mail address: robles@math.tamu.edu

RECEIVED OCTOBER 22, 2012 
